\title{
CONVEX DUALITY IN CONSTRAINED MEAN-VARIANCE PORTFOLIO OPTIMIZATION
}

\author{
CHANTAL LABBÉ,**** HEC Montréal \\ ANDREW J. HEUNIS, ${ }^{* * * * *}$ University of Waterloo
}

\begin{abstract}
We apply conjugate duality to establish the existence of optimal portfolios in an assetallocation problem, with the goal of minimizing the variance of the final wealth which results from trading over a fixed, finite horizon in a continuous-time, complete market, subject to the constraints that the expected final wealth equal a specified target value and the portfolio of the investor (defined by the dollar amount invested in each stock) take values in a given closed, convex set. The asset prices are modelled by Itô processes, for which the market parameters are random processes adapted to the information filtration available to the investor. We synthesize a dual optimization problem and establish a set of optimality relations, similar to the Euler-Lagrange and transversality relations of calculus of variations, giving necessary and sufficient conditions for the given optimization problem and its dual to each have a solution, with zero duality gap. We then solve these relations, to establish the existence of an optimal portfolio.
\end{abstract}

Keywords: Convex analysis; duality synthesis; variational analysis

2000 Mathematics Subject Classification: Primary 93E20; 91B28; 90A09; 49N15

\section{Introduction}

In this work we study an asset-allocation problem the goal of which is to minimize the variance of the final wealth which results from trading on a fixed, finite horizon in a continuoustime, complete market with random market parameters, subject to the constraints that the expected final wealth equal a specified target value and the portfolio of the investor (defined by the dollar amount in each stock) always take values in a given closed, convex set. This constraint is general enough to model a prohibition on the short selling of stock, incomplete markets, limits on the dollar amount allocated to each stock, and other trading restrictions. Our goal is to establish the existence of an optimal portfolio and then characterize it.

Problems of this kind belong to the general area of mean-variance portfolio selection, and their financial relevance, as compared with that of the more common problem of maximizing expected utility, has been discussed by Lim and Zhou [13] and Li et al. [12]. Lim and Zhou [13] addressed mean-variance portfolio selection with unconstrained portfolios, using the methods of stochastic linear quadratic control. The follow-up work [12] deals with a similar problem, but includes a no-short selling constraint; it was postulated that the market coefficients are nonrandom, and viscosity solutions to the (correspondingly nonrandom) Bellman equation were used to characterize the constrained optimal portfolio. The problem of interest here

Received 17 August 2004; revision received 12 November 2006.

* Postal address: HEC Montréal, Montréal, QC H3T 2A7, Canada. Email address: chantal.labbe@ hec.ca

** Postal address: Department of Electrical and Computer Engineering, University of Waterloo, Waterloo, ON N2L 3G1, Canada. Email address: heunis@kingcong.uwaterloo.ca

*** Supported by the National Sciences and Engineering Research Council of Canada. 
involves a combination of both random market parameters and general portfolio constraints. This rules out application of stochastic linear quadratic theory, as in [13] (which relies on the absence of portfolio constraints), as well as the approach of [12] (for which the market parameters must be nonrandom).

In light of this, we turn to the use of conjugate duality. The goal is to formulate an associated 'dual' optimization problem for which it is (hopefully) easy to establish the existence of a solution directly, and then to construct an optimal portfolio in terms of the solution to the dual problem. Our approach is motivated by a recent work of Rogers, [14], in which the central idea is to regard the dynamical relation satisfied by the wealth and the portfolio as itself defining $a$ constraint, a point of view which then provides the key for synthesizing a dual optimization problem. We cannot in fact directly apply the method of [14], since that work does not address the problem of the existence of optimal portfolios; nevertheless, the fundamental viewpoint of [14], namely that the wealth equation is a constraint, is essential to us. We shall account for this constraint in a way which is suggested by a work of Bismut, [2], on stochastic convex control problems. The basic idea is to remove the portfolio 'variable' to obtain a Bolza problem in (stochastic) calculus of variations which amounts to the minimization of a convex functional over a set of Itô processes large enough to include all of the possible wealth processes. Bismut [2] established a powerful duality theory for dealing with such stochastic Bolza problems, and we shall use it to construct a dual optimization problem together with optimality relations (similar to the Euler-Lagrange and transversality relations of calculus of variations) which are equivalent to the primal and dual problems being solvable with zero duality gap. We then use these relations to establish the existence of an optimal portfolio and the corresponding wealth process.

In Sections 2-4 we introduce the market model and formulate the problem of constrained mean-variance portfolio selection, and in Sections 5 and 6 we use conjugate duality to construct the optimal portfolio and wealth process. Finally, in Section 7 we indicate how the approach to mean-variance minimization that we have used extends easily to problems of utility maximization.

\section{Market model}

Throughout the paper, $T \in(0, \infty)$ denotes a given constant and $\{\boldsymbol{W}(t), t \in[0, T]\}$ is a given $\mathbb{R}^{N}$-valued standard Brownian motion, with scalar entries $W_{m}(t), m=1,2, \ldots, N$, on the complete probability space $(\Omega, \mathcal{F}, \mathrm{P})$. Let

$$
\mathcal{F}_{t}:=\sigma\{\boldsymbol{W}(\tau), \tau \in[0, t]\} \vee \mathcal{N}(\mathrm{P}),
$$

in which $\mathcal{N}(\mathrm{P})$ denotes the collection of all P-null events in $(\Omega, \mathcal{F}, \mathrm{P})$. We consider a market comprising $N+1$ assets traded continuously on the interval $[0, T]$, namely a bond with price $\left\{S_{0}(t)\right\}$ given by

$$
\mathrm{d} S_{0}(t)=r(t) S_{0}(t) \mathrm{d} t, \quad 0 \leq t \leq T, \quad S_{0}(0)=1,
$$

and $N$ stocks with prices $\left\{S_{n}(t)\right\}, n=1,2, \ldots, N$, given by

$$
\mathrm{d} S_{n}(t)=S_{n}(t)\left[b_{n}(t) \mathrm{d} t+\sum_{m=1}^{N} \sigma_{n m}(t) \mathrm{d} W_{m}(t)\right], \quad 0 \leq t \leq T,
$$

the initial values $S_{n}(0)$ being given strictly positive constants. From now on we shall assume the following condition. 
Condition 2.1. In (2.2) and (2.3) the interest rate, $\{r(t)\}$, the entries $\left\{b_{n}(t)\right\}$ of $\{\boldsymbol{b}(t)\}$, the $\mathbb{R}^{N}$-valued process of mean rates of return on stocks, and the entries $\left\{\sigma_{n m}(t)\right\}$ of $\{\sigma(t)\}$, the $N \times N$ matrix-valued volatility process are uniformly bounded and $\left\{\mathcal{F}_{t}\right\}$-progressively measurable scalar processes on $\Omega \times[0, T]$; furthermore, $\{r(t)\}$ is nonnegative. There exists a constant $\kappa \in(0, \infty)$ such that $\boldsymbol{z}^{\top} \boldsymbol{\sigma}(\omega, t) \boldsymbol{\sigma}^{\top}(\omega, t) \boldsymbol{z} \geq \kappa\|\boldsymbol{z}\|^{2}$ for all $(z, \omega, t) \in \mathbb{R}^{N} \times \Omega \times[0, T]$. (We use $\|z\|$ to denote the usual Euclidean length of a vector $z \in \mathbb{R}^{N}$.)

Remark 2.1. In view of Condition 2.1 and [8, Problem 5.8.1, p. 372], there exists a constant $\kappa_{1} \in(0, \infty)$ such that $\max \left\{\left\|\boldsymbol{\sigma}^{-1}(\omega, t) z\right\|,\left\|\left[\boldsymbol{\sigma}^{\top}\right]^{-1}(\omega, t) z\right\|\right\} \leq \kappa_{1}\|z\|$ for all $(z, \omega, t) \in \mathbb{R}^{N} \times$ $\Omega \times[0, T]$. This bound will often be used.

Remark 2.2. Define the usual market price of risk,

$$
\boldsymbol{\theta}(t):=\boldsymbol{\sigma}^{-1}(t)[\boldsymbol{b}(t)-r(t) \mathbf{1}]
$$

in which $\mathbf{1} \in \mathbb{R}^{N}$ has all unit entries. From Condition 2.1 and Remark 2.1, we see that $\{\boldsymbol{\theta}(t)\}$ is uniformly bounded on $\Omega \times[0, T]$.

Given some $x_{0} \in \mathbb{R}$ and some $\left\{\mathcal{F}_{t}\right\}$-progressively measurable process $\pi: \Omega \times[0, T] \rightarrow \mathbb{R}^{N}$ satisfying $\int_{0}^{T}\|\pi(t)\|^{2} \mathrm{~d} t<\infty$ almost surely (a.s.), it follows that there exists a scalar-valued, continuous, $\left\{\mathscr{F}_{t}\right\}$-progressively measurable process $\left\{X^{\pi}(t), t \in[0, T]\right\}$ such that

$$
\mathrm{d} X^{\pi}(t)=\left\{r(t) X^{\pi}(t)+\boldsymbol{\pi}^{\top}(t) \boldsymbol{\sigma}(t) \boldsymbol{\theta}(t)\right\} \mathrm{d} t+\boldsymbol{\pi}^{\top}(t) \boldsymbol{\sigma}(t) \mathrm{d} \boldsymbol{W}(t), \quad X^{\pi}(0)=x_{0} .
$$

This process is unique (up to indistinguishability) and is given by

$$
X^{\pi}(t)=S_{0}(t)\left\{x_{0}+\int_{0}^{t} S_{0}^{-1}(\tau) \boldsymbol{\pi}^{\top}(\tau) \boldsymbol{\sigma}(\tau) \boldsymbol{\theta}(\tau) \mathrm{d} \tau+\int_{0}^{t} S_{0}^{-1}(\tau) \boldsymbol{\pi}^{\top}(\tau) \boldsymbol{\sigma}(\tau) \mathrm{d} \boldsymbol{W}(\tau)\right\} .
$$

From now on we consider a small investor who trades in the market following a self-funded strategy and has a given initial wealth $x_{0} \in(0, \infty)$. If $\pi_{n}(t)$, the $n$th entry of the $\mathbb{R}^{N}$-valued vector $\pi(t)$, is interpreted as the dollar amount invested in the stock with price $S_{n}(t)$, for $n=1,2, \ldots, N$, then it follows from (2.2), (2.3), and [9, Remark 1.3.3] that $X^{\pi}(t)$ gives the investor's wealth at instant $t \in[0, T]$.

\section{A class of square-integrable Itô processes}

We formulate the optimization problem in this section, but must first define a class of squareintegrable Itô processes which will be essential in all later developments.

We write $\mathcal{F}^{*}$ for the $\left\{\mathcal{F}_{t}\right\}$-progressively measurable $\sigma$-algebra on $\Omega \times[0, T]$. The measure space $\left(\Omega \times[0, T], \mathcal{F}^{*},(\mathrm{P} \otimes \lambda)\right.$ ), where $\lambda$ stands for the Lebesgue measure (on the Borel $\sigma$-algebra on $[0, T])$, is used extensively, and the qualifier 'a.e.' always refers to the measure $(\mathrm{P} \otimes \lambda)$ on $\Omega \times[0, T]$. For example, if $\pi$ is an $\mathbb{R}^{N}$-valued, $\left\{\mathcal{F}^{*}\right\}$-measurable mapping on $\Omega \times[0, T]$ and $K \subset \mathbb{R}^{N}$, then $\pi(t) \in K$ a.e. means that $\pi(\omega, t) \in K$ for $(\mathrm{P} \otimes \lambda)$-almost all $(\omega, t)$. Similarly, the qualifier 'a.s.' is always used with reference to the probability measure $\mathrm{P}$ on $\mathcal{F}$. For any mapping $\xi$ on $\Omega \times[0, T]$ with values in some Euclidean space (the dimensionality of which will be clear from the context) we write $\xi \in \mathcal{F}^{*}$ to indicate that $\xi$ is $\left\{\mathcal{F}^{*}\right\}$-measurable. 
Motivated by [2, p. 386, p. 390], we let

$$
\begin{aligned}
L_{21} & :=\left\{v: \Omega \times[0, T] \rightarrow \mathbb{R} \text { such that } v \in \mathcal{F}^{*} \text { and } \mathrm{E}\left[\left(\int_{0}^{T}|v(t)| \mathrm{d} t\right)^{2}\right]<\infty\right\}, \\
L_{22} & :=\left\{\xi: \Omega \times[0, T] \rightarrow \mathbb{R}^{N} \text { such that } \xi \in \mathcal{F}^{*} \text { and } \mathrm{E}\left[\int_{0}^{T}\|\xi(t)\|^{2} \mathrm{~d} t\right]<\infty\right\}, \\
\mathbb{B} & :=\mathbb{R} \times L_{21} \times L_{22} .
\end{aligned}
$$

We write $X \in \mathbb{B}$ to indicate that $\left\{\left(X(t), \mathcal{F}_{t}\right), t \in[0, T]\right\}$ is a continuous semimartingale of the form

$$
X(t)=X_{0}+\int_{0}^{t} \dot{X}(\tau) \mathrm{d} \tau+\int_{0}^{t} \boldsymbol{\Lambda}_{X}^{\top}(\tau) \mathrm{d} \boldsymbol{W}(\tau),
$$

for some $\left(X_{0}, \dot{X}, \boldsymbol{\Lambda}_{X}\right) \in \mathbb{B}$, and write $X \equiv\left(X_{0}, \dot{X}, \boldsymbol{\Lambda}_{X}\right)$ to indicate that (3.1) holds. In expansion (3.1) it is clear that the integrands $\dot{X}$ and $\Lambda_{X}$ are uniquely determined a.e. on $\Omega \times$ $[0, T]$. The set $\mathbb{B}$ is essentially the collection of all square-integrable Itô processes with respect to the Brownian motion $\{\boldsymbol{W}(t)\}$. From Doob's $L^{2}$-inequality we immediately have

$$
\mathrm{E}\left[\sup _{0 \leq t \leq T}|X(t)|^{2}\right]<\infty \quad \text { for } X \in \mathbb{B} .
$$

Note from (2.4) that, for any given $\mathbb{R}^{N}$-valued $\pi \in \mathcal{F}^{*}$ for which the stochastic integration is defined, $X^{\pi}$ is an Itô process with respect to the Brownian motion $\{\boldsymbol{W}(t)\}$. The next result gives conditions on $\pi$ for membership of $X^{\pi}$ in $\mathbb{B}$. The proof is elementary and is omitted.

Proposition 3.1. Assume that Condition 2.1 is satisfied, and suppose that $\pi: \Omega \times[0, T] \rightarrow \mathbb{R}^{N}$ is $\left\{\mathcal{F}^{*}\right\}$-measurable and that $\int_{0}^{T}\|\pi(t)\|^{2} \mathrm{~d} t<\infty$ a.s. Then $X^{\pi} \in \mathbb{B}$ if and only if $\pi \in L_{22}$.

\section{The optimization problem}

In order to formulate the optimization problem we impose the following basic conditions.

Condition 4.1. We are given a closed, convex set $K \subset \mathbb{R}^{N}$ with $\mathbf{0} \in K$ and an $\left\{\mathcal{F}_{T}\right\}$-measurable random variable a on $(\Omega, \mathcal{F}, \mathrm{P})$ such that $0<\inf _{\omega \in \Omega} a(\omega) \leq \sup _{\omega \in \Omega} a(\omega)<\infty$.

Condition 4.2. We are given a number $d \in \mathbb{R}$ and $\left\{\mathcal{F}_{T}\right\}$-measurable, square-integrable random variables $c_{0}$ and $c_{1}$ on $(\Omega, \mathcal{F}, \mathrm{P})$.

Let

$$
\begin{gathered}
\mathcal{A}:=\left\{\pi \in L_{22}: \pi(t) \in K \text { a.e. }\right\}, \\
\hat{J}(\omega, x):=\frac{1}{2}\left[a(\omega) x^{2}+2 c_{0}(\omega) x\right], \quad(\omega, x) \in \Omega \times \mathbb{R}, \\
G(\pi):=\mathrm{E}\left[c_{1} X^{\pi}(T)\right]-d, \quad \pi \in L_{22}, \\
\hat{\vartheta}:=\inf _{\substack{\boldsymbol{\pi} \in \mathcal{A} \\
G(\pi)=0}} \mathrm{E}\left[\hat{J}\left(X^{\pi}(T)\right)\right] .
\end{gathered}
$$

We regard $\mathcal{A}$ as the set of admissible portfolios, while $G(\boldsymbol{\pi})=0$ represents a constraint on the terminal wealth. The problem of interest, which we denote by $(\hat{\mathcal{P}})$, is to

$$
\text { determine some } \hat{\pi} \in \mathcal{A} \text { such that } G(\hat{\pi})=0 \text { and } \hat{\vartheta}=\mathrm{E}\left[\hat{J}\left(X^{\hat{\pi}}(T)\right)\right] \text {, }
$$


in the sense of demonstrating the existence of $\hat{\boldsymbol{\pi}}$ and characterizing its dependence on the market parameters $\{r(t)\},\{\boldsymbol{b}(t)\}$, and $\{\boldsymbol{\sigma}(t)\}$ and $\left\{\mathcal{F}_{t}\right\}$, the information filtration available to the investor. We must also postulate that $0 \in\{G(\pi): \pi \in \mathcal{A}\}$, since otherwise the constraints on $\pi$ in (4.4) are mutually contradictory and we will have $\hat{\vartheta}=\infty$, rendering problem (4.5) meaningless. In fact, we impose the following constraint qualification.

Condition 4.3. The constant $d$, the set $K$, and the random variable $c_{1}$ are such that the set $\{G(\pi): \pi \in \mathcal{A}\} \subset \mathbb{R}$ has a nonempty interior which includes 0 (see Remark 4.2).

Example 4.1. In (4.1), $K=\mathbb{R}^{N}$ corresponds to there being no constraints on the portfolio. On the other hand, $K=[0, \infty)^{N}$ represents a short selling prohibition on stocks, while the constraint set $K=\left\{\pi \in[0, \infty)^{N}: \pi_{n+1}=\cdots=\pi_{N}=0\right\}$ represents the same prohibition, but in an incomplete market for which $N$, the dimension of the Brownian motion $\{\boldsymbol{W}(t)\}$, exceeds $n$, the number of stocks available to the investor. Other examples can be similarly formulated.

Remark 4.1. The most important case of problem (4.5) occurs when $a=2, c_{0}=0$, and $c_{1}=1$, since then $\mathrm{E}\left[\hat{J}\left(X^{\pi}(T)\right)\right]-d^{2}=\operatorname{var}\left(X^{\pi}(T)\right)$ (the variance of the terminal wealth) if $G(\pi)=0$. Problem (4.5) amounts to minimizing this variance subject to the terminal wealth constraint $\mathrm{E}\left[X^{\pi}(T)\right]=d$ together with the portfolio constraint $\pi \in \mathcal{A}$. This is the problem of constrained mean-variance portfolio selection.

Remark 4.2. We observe that Condition 4.3 holds in the case in which $c_{1} \equiv 1$ in (4.3), the market model is 'interesting' in the sense that $\mathrm{E}\left[X^{\tilde{\pi}}(T)\right]>\mathrm{E}\left[x_{0} S_{0}(T)\right]$ for some portfolio $\tilde{\boldsymbol{\pi}} \in \mathcal{A}$ (problem (4.5) would be pointless otherwise, since the best expected terminal wealth would be attained by simply investing the entire fortune, risk free, in the money market), and the expected terminal wealth, $d$, in (4.3) is 'reasonable' in a sense to be specified. From (4.1), the convexity of $K \subset \mathbb{R}^{N}$ (see Condition 4.1), and (2.5), it follows that $\mathcal{R}:=\left\{\mathrm{E}\left[X^{\pi}(T)\right]: \pi \in\right.$ $\mathcal{A}\} \subset \mathbb{R}$ is convex and, hence, an interval. Thus, the interior of $\mathcal{R}$ is identical to $\mathcal{I}:=$ $\operatorname{(inf}_{\boldsymbol{\pi} \in \mathcal{A}} \mathrm{E}\left[X^{\pi}(T)\right], \sup _{\pi \in \mathcal{A}} \mathrm{E}\left[X^{\pi}(T)\right]$ ), which is nonempty since $\mathbf{0} \in K$ and the market model is interesting in the above sense. It follows from (4.3) that Condition 4.3 holds provided that $d$ is specified in the 'reasonable range' $\mathcal{I}$, that is, $d \in \mathcal{I}$.

\section{A partially constrained problem}

Here we establish duality relations for a partially constrained optimization problem in which the terminal wealth condition, $G(\boldsymbol{\pi})=0$, of (4.5) is discarded. In Section 6 these relations will be used to establish the existence of a solution to the fully constrained problem. We postulate the following condition.

Condition 5.1. We are given a constant $q \in \mathbb{R}$ and an $\left\{\mathcal{F}_{T}\right\}$-measurable, square-integrable random variable c on $(\Omega, \mathcal{F}, \mathrm{P})$.

Recalling the random variable $a$ and the convex set $K$ assumed given in Condition 4.1, and the set $\mathcal{A}$ defined in (4.1), we let

$$
\vartheta_{c, q}:=\inf _{\pi \in \mathcal{A}} \mathrm{E}\left[J\left(X^{\pi}(T)\right)\right], \quad \text { where } J(\omega, x):=\frac{1}{2}\left[a(\omega) x^{2}+2 c(\omega) x\right]+q .
$$

The partially constrained optimization problem, which we denote by $\left(\mathcal{P}_{c, q}\right)$, is to

$$
\text { determine some } \overline{\boldsymbol{\pi}} \in \mathcal{A} \text { such that } \vartheta_{c, q}=\mathrm{E}\left[J\left(X^{\bar{\pi}}(T)\right)\right] \text {. }
$$


Remark 5.1. We distinguish between the respective coefficients $c_{0}$ and $c$ in the linear terms of $\hat{J}$ and $J$ (see (4.2) and (5.1)) because in Section 6 these coefficients will play somewhat different roles. From the quadratic form of $x \mapsto J(\omega, x)$ in (5.1), Conditions 4.1 and 5.1, and Proposition 3.1, it follows immediately that $-\infty<\vartheta_{c, q}<\infty$.

\subsection{Synthesis of a dual problem and optimality relations}

Our goals are to reformulate problem (5.2) as a 'primal' optimization problem over the set $\mathbb{B}$ of Section 3 (see (5.11)), synthesize a dual optimization problem and corresponding EulerLagrange-Hamilton optimality relations (see Proposition 5.3) by following an algorithmic approach motivated in [2], and establish the existence of a solution to the dual problem (see Proposition 5.4).

Step I. From Proposition 3.1 we know that $X^{\pi} \in \mathbb{B}$ for each admissible $\pi \in \mathcal{A}$. We therefore express the quantity in (5.1) as the infimum over the set $\mathbb{B}$ of some appropriate mapping $\Phi: \mathbb{B} \rightarrow(-\infty, \infty]$, by introducing 'penalty terms' on $\mathbb{B}$ which account for the initial-wealth constraint, $X(0)=x_{0}$; the portfolio constraint, $\pi(t) \in K$ a.e.; and the 'dynamical constraint' implicit in (2.4). These will be defined to give zero penalty when the constraints hold and infinite penalty otherwise. For each $X \equiv\left(X_{0}, \dot{X}, \boldsymbol{\Lambda}_{X}\right) \in \mathbb{B}$, we let

$$
\mathcal{U}(X):=\left\{\boldsymbol{\pi} \in \mathcal{A}: \dot{X}(t)=r(t) X(t)+\boldsymbol{\pi}^{\top}(t) \boldsymbol{\sigma}(t) \boldsymbol{\theta}(t) \text { and } \boldsymbol{\Lambda}_{X}(t)=\boldsymbol{\sigma}^{\top}(t) \boldsymbol{\pi}(t) \text { a.e. }\right\} .
$$

We then see that for each $X \equiv\left(X_{0}, \dot{X}, \boldsymbol{\Lambda}_{X}\right) \in \mathbb{B}$ we have $X(t)=X^{\pi}(t)$ a.e. for some $\pi \in \mathcal{A}$ if and only if $X_{0}=x_{0}$ and $\mathcal{U}(X) \neq \varnothing$; from this equivalence and (5.1), we obtain

$$
\vartheta_{c, q}=\inf _{\substack{X \in \mathbb{B}, X_{0}=x_{0} \\ U(X) \neq \varnothing}} \mathrm{E}[J(X(T))]
$$

We now define a penalty function on $\mathbb{B}$ giving zero penalty when the constraint $\mathcal{U}(X) \neq \varnothing$ is satisfied and infinite penalty otherwise. From Remark 2.1 , for each $X \equiv\left(X_{0}, \dot{X}, \boldsymbol{\Lambda}_{X}\right) \in \mathbb{B}$,

$$
\begin{aligned}
\mathcal{U}(X) \neq \varnothing \quad & \quad \dot{X}(t)=r(t) X(t)+\boldsymbol{\Lambda}_{X}^{\top}(t) \boldsymbol{\theta}(t) \\
& \text { and }\left[\boldsymbol{\sigma}^{\top}\right]^{-1}(t) \boldsymbol{\Lambda}_{X}(t) \in K \quad \text { a.e. }
\end{aligned}
$$

Motivated by (5.5), we define the mapping $L: \Omega \times[0, T] \times \mathbb{R} \times \mathbb{R} \times \mathbb{R}^{N} \rightarrow\{0, \infty\}$ by

$$
L(\omega, t, x, v, \xi)= \begin{cases}0 & \text { if } v=r(\omega, t) x+\boldsymbol{\xi}^{\top} \boldsymbol{\theta}(\omega, t) \text { and }\left[\boldsymbol{\sigma}^{\top}\right]^{-1}(\omega, t) \xi \in K, \\ \infty & \text { otherwise. }\end{cases}
$$

It is clear that $L\left(t, X(t), \dot{X}(t), \boldsymbol{\Lambda}_{X}(t)\right)$ is $\left\{\mathcal{F}^{*}\right\}$-measurable, and, in view of (5.5) and (5.6),

$$
\mathrm{E}\left[\int_{0}^{T} L\left(t, X(t), \dot{X}(t), \boldsymbol{\Lambda}_{X}(t)\right) \mathrm{d} t\right]= \begin{cases}0 & \text { if } \mathcal{U}(X) \neq \varnothing \\ \infty & \text { otherwise }\end{cases}
$$

for each $X \in \mathbb{B}$. We see that (5.7) establishes a penalty for the constraint $\mathcal{U}(X) \neq \varnothing$ in (5.4). As for the initial-wealth constraint, $X_{0}=x_{0}$, we let

$$
l_{0}(x):= \begin{cases}0 & \text { if } x=x_{0} \\ \infty & \text { otherwise }\end{cases}
$$


for each $x \in \mathbb{R}$. Now define

$$
\Phi(X):=l_{0}\left(X_{0}\right)+\mathrm{E}\left[l_{T}(X(T))\right]+\mathrm{E}\left[\int_{0}^{T} L\left(t, X(t), \dot{X}(t), \Lambda_{X}(t)\right) \mathrm{d} t\right]
$$

for each $X \equiv\left(X_{0}, \dot{X}, \boldsymbol{\Lambda}_{X}\right) \in \mathbb{B}$, where, for consistency of notation, we let

$$
l_{T}(\omega, x):=J(\omega, x), \quad(\omega, x) \in \Omega \times \mathbb{R} .
$$

Upon combining (5.4) and (5.7)-(5.10), we obtain

$$
\vartheta_{c, q}=\inf _{X \in \mathbb{B}} \Phi(X) .
$$

Remark 5.2. From (5.7) and (5.8) it is clear that $\Phi(X)$ exists in $(-\infty, \infty]$ for each $X \in \mathbb{B}$.

Step II. In this step we synthesize a 'cost' functional $\Psi: \mathbb{B} \rightarrow(-\infty, \infty]$ for an optimization problem which is dual to the primal problem (5.11). To this end we define the convex conjugate functions

$$
\begin{aligned}
m_{0}(y):=l_{0}^{*}(y) & :=\sup _{x \in \mathbb{R}}\left\{x y-l_{0}(x)\right\}, \\
m_{T}(\omega, y):=l_{T}^{*}(\omega,-y) & :=\sup _{x \in \mathbb{R}}\left\{x(-y)-l_{T}(\omega, x)\right\}, \\
M(\omega, t, y, s, \boldsymbol{\gamma}):=L^{*}(\omega, t, s, y, \boldsymbol{\gamma}):= & \sup _{\substack{x, v \in \mathbb{R} \\
\xi \in \mathbb{R}^{N}}}\left\{x s+v y+\boldsymbol{\xi}^{\top} \boldsymbol{\gamma}-L(\omega, t, x, v, \boldsymbol{\xi})\right\},
\end{aligned}
$$

for each $y \in \mathbb{R}, s \in \mathbb{R}, \boldsymbol{\gamma} \in \mathbb{R}^{N}, \omega \in \Omega$, and $t \in[0, T]$. From (5.8), (5.10), and (5.6), it is easy to calculate these conjugates explicitly: for each $(\omega, y) \in \Omega \times \mathbb{R}$, we have

$$
\begin{gathered}
m_{0}(y)=x_{0} y, \quad m_{T}(\omega, y)=\frac{(y+c(\omega))^{2}}{2 a(\omega)}-q, \\
M(\omega, t, y, s, \boldsymbol{\gamma})= \begin{cases}\delta(-\boldsymbol{\sigma}(t)[\boldsymbol{\theta}(t) y+\boldsymbol{\gamma}]) & \text { if } s+r(t) y=0, \\
\infty & \text { otherwise }\end{cases}
\end{gathered}
$$

where $\delta(\cdot)$ is the support functional of the set $-K$, defined by

$$
\delta(z):=\sup _{\pi \in K}\left\{-\pi^{\top} z\right\}, \quad z \in \mathbb{R}^{N} .
$$

For each $Y \equiv\left(Y_{0}, \dot{Y}, \boldsymbol{\Lambda}_{Y}\right) \in \mathbb{B}$, define

$$
\Psi(Y):=m_{0}\left(Y_{0}\right)+\mathrm{E}\left[m_{T}(Y(T))\right]+\mathrm{E}\left[\int_{0}^{T} M\left(t, Y(t), \dot{Y}(t), \Lambda_{Y}(t)\right) \mathrm{d} t\right] .
$$

Remark 5.3. Since $\delta(\cdot)$ is lower semicontinuous on $\mathbb{R}^{N}$, it is easily seen from (5.14) that $M\left(t, Y(t), \dot{Y}(t), \boldsymbol{\Lambda}_{Y}(t)\right)$ is $\left\{\mathcal{F}^{*}\right\}$-measurable for each $Y \equiv\left(Y_{0}, \dot{Y}, \boldsymbol{\Lambda}_{Y}\right) \in \mathbb{B}$, and it is clear that $\Psi(Y)$ exists in $(-\infty, \infty]$ for each $Y \in \mathbb{B}$.

Next we require the following result, which is [2, Proposition I-1]. 
Proposition 5.1. For members $X \equiv\left(X_{0}, \dot{X}, \boldsymbol{\Lambda}_{X}\right)$ and $Y \equiv\left(Y_{0}, \dot{Y}, \boldsymbol{\Lambda}_{Y}\right)$ of the set $\mathbb{B}$, define

$$
\begin{array}{r}
\mathbb{M}(X, Y)(t):=X(t) Y(t)-X_{0} Y_{0}-\int_{0}^{t}\left\{X(\tau) \dot{Y}(\tau)+\dot{X}(\tau) Y(\tau)+\Lambda_{X}^{\top}(\tau) \boldsymbol{\Lambda}_{Y}(\tau)\right\} \mathrm{d} \tau \\
t \in[0, T] .
\end{array}
$$

Then $\left\{\left(\mathbb{M}(X, Y)(t), \mathcal{F}_{t}\right), t \in[0, T]\right\}$ is a continuous martingale with $\mathbb{M}(X, Y)(0)=0$.

Proposition 5.2. Assume that Conditions 2.1, 4.1, and 5.1 are satisfied. Then the functions $\Phi$ and $\Psi$ respectively given in (5.9) and (5.16) are well defined, take values in $(-\infty, \infty]$ for each $X \in \mathbb{B}$ and $Y \in \mathbb{B}$, and satisfy

$$
\Phi(X)+\Psi(Y) \geq 0, \quad(X, Y) \in \mathbb{B} \times \mathbb{B} .
$$

Moreover, for arbitrary $\bar{X} \equiv\left(\bar{X}_{0}, \dot{\bar{X}}, \boldsymbol{\Lambda}_{\bar{X}}\right) \in \mathbb{B}$ and $\bar{Y} \equiv\left(\bar{Y}_{0}, \dot{\bar{Y}}, \boldsymbol{\Lambda}_{\bar{Y}}\right) \in \mathbb{B}$, we have $\Phi(\bar{X})+$ $\Psi(\bar{Y})=0$ if and only if each of the following conditions hold:

$$
\begin{gathered}
l_{0}\left(\bar{X}_{0}\right)+m_{0}\left(\bar{Y}_{0}\right)=\bar{X}_{0} \bar{Y}_{0}, \\
l_{T}(\bar{X}(T))+m_{T}(\bar{Y}(T))=-\bar{X}(T) \bar{Y}(T) \quad \text { a.s. }, \\
L\left(t, \bar{X}(t), \dot{\bar{X}}(t), \boldsymbol{\Lambda}_{\bar{X}}(t)\right)+M\left(t, \bar{Y}(t), \dot{\bar{Y}}(t), \Lambda_{\bar{Y}}(t)\right) \\
=\bar{X}(t) \dot{\bar{Y}}(t)+\dot{\bar{X}}(t) \bar{Y}(t)+\boldsymbol{\Lambda}_{\bar{X}}^{\top}(t) \Lambda_{\bar{Y}}(t) \quad \text { a.e. }
\end{gathered}
$$

Proof. Fix $X \equiv\left(X_{0}, \dot{X}, \boldsymbol{\Lambda}_{X}\right) \in \mathbb{B}$ and $Y \equiv\left(Y_{0}, \dot{Y}, \boldsymbol{\Lambda}_{Y}\right) \in \mathbb{B}$. To establish (5.17), observe from the convex conjugates in (5.12) that, for each $(\omega, t) \in \Omega \times[0, T]$,

$$
\begin{gathered}
l_{0}\left(X_{0}\right)+m_{0}\left(Y_{0}\right) \geq X_{0} Y_{0}, \\
l_{T}(X(T))+m_{T}(Y(T)) \geq-X(T) Y(T), \\
L\left(t, X(t), \dot{X}(t), \boldsymbol{\Lambda}_{X}(t)\right)+M\left(t, Y(t), \dot{Y}(t), \boldsymbol{\Lambda}_{Y}(t)\right) \\
\geq X(t) \dot{Y}(t)+\dot{X}(t) Y(t)+\boldsymbol{\Lambda}_{X}^{\top}(t) \boldsymbol{\Lambda}_{Y}(t) .
\end{gathered}
$$

By (5.9), (5.16), (5.21), and the definition of $\mathbb{M}(X, Y)$ (see Proposition 5.1),

$$
\begin{aligned}
\Phi(X)+\Psi(Y)= & l_{0}\left(X_{0}\right)+m_{0}\left(Y_{0}\right)+\mathrm{E}\left[l_{T}(X(T))+m_{T}(Y(T))\right] \\
& +\mathrm{E}\left[\int_{0}^{T}\left\{L\left(t, X(t), \dot{X}(t), \boldsymbol{\Lambda}_{X}(t)\right)+M\left(t, Y(t), \dot{Y}(t), \boldsymbol{\Lambda}_{Y}(t)\right)\right\} \mathrm{d} t\right] \\
\geq & X_{0} Y_{0}+\mathrm{E}[-X(T) Y(T)] \\
& +\mathrm{E}\left[\int_{0}^{T}\left\{X(t) \dot{Y}(t)+\dot{X}(t) Y(t)+\boldsymbol{\Lambda}_{X}^{\top}(t) \boldsymbol{\Lambda}_{Y}(t)\right\} \mathrm{d} t\right] \\
= & \mathrm{E}[-\mathbb{M}(X, Y)(T)] .
\end{aligned}
$$

Moreover, $\mathrm{E}[-\mathbb{M}(X, Y)(T)]=0$ (from Proposition 5.1), which establishes (5.17). Furthermore, for some $(\bar{X}, \bar{Y}) \in \mathbb{B} \times \mathbb{B}$ the equivalence between $\Phi(\bar{X})+\Psi(\bar{Y})=0$ and (5.18)-(5.20) follows at once from (5.22), (5.21), and the fact that $\mathrm{E}[-\mathbb{M}(X, Y)(T)]=0$.

We now refine Proposition 5.2, to obtain Proposition 5.3, which gives a set of optimality relations that will be essential in constructing an optimal portfolio. Let

$$
\boldsymbol{\Theta}_{Y}(t):=-\boldsymbol{\sigma}(t)\left[\boldsymbol{\theta}(t) Y(t)+\boldsymbol{\Lambda}_{Y}(t)\right] \text { for } Y \equiv\left(Y_{0}, \dot{Y}, \boldsymbol{\Lambda}_{Y}\right) \in \mathbb{B} .
$$


Proposition 5.3. Assume that Conditions 2.1, 4.1, and 5.1 are satisfied. Then, for an arbitrary $(\bar{X}, \bar{Y}) \in \mathbb{B} \times \mathbb{B}$, we have

$$
\Phi(\bar{X})=\vartheta_{c, q}=\sup _{Y \in \mathbb{B}}\{-\Psi(Y)\}=-\Psi(\bar{Y})
$$

if and only if

$$
\begin{gathered}
\bar{X}_{0}=x_{0}, \\
\bar{X}(T)=-\frac{\bar{Y}(T)+c}{a} \quad \text { a.s. } \\
\dot{\bar{Y}}(t)+r(t) \bar{Y}(t)=0 \quad \text { a.e. } \\
\bar{\pi} \in \mathcal{U}(\bar{X}) \text { and } \delta\left(\boldsymbol{\Theta}_{\bar{Y}}(t)\right)+\bar{\pi}^{\top}(t) \Theta_{\bar{Y}}(t)=0 \quad \text { a.e. } \\
\text { for } \overline{\boldsymbol{\pi}}(t):=\left[\sigma^{\top}\right]^{-1}(t) \boldsymbol{\Lambda}_{\bar{X}}(t) .
\end{gathered}
$$

Proof. From (5.6) and (5.14), for arbitrary $(\omega, t) \in \Omega \times[0, T]$ and $(x, v, \xi),(y, s, \boldsymbol{\gamma}) \in$ $\mathbb{R} \times \mathbb{R} \times \mathbb{R}^{N}$ we have the equivalence

$$
\begin{aligned}
& L(\omega, t, x, v, \boldsymbol{\xi})+M(\omega, t, y, s, \boldsymbol{\gamma})=x s+v y+\boldsymbol{\xi}^{\top} \boldsymbol{\gamma} \\
& \Longleftrightarrow \quad \begin{array}{r}
v=r(t) x+\boldsymbol{\xi}^{\top} \boldsymbol{\theta}(t), \quad\left[\boldsymbol{\sigma}^{\top}\right]^{-1}(t) \boldsymbol{\xi} \in K, \quad s+r(t) y=0, \\
\quad \text { and } \delta(-\boldsymbol{\sigma}(t)[\boldsymbol{\theta}(t) y+\boldsymbol{\gamma}])-\boldsymbol{\xi}^{\top} \boldsymbol{\sigma}^{-1}(t) \boldsymbol{\sigma}(t)[\boldsymbol{\theta}(t) y+\boldsymbol{\gamma}]=0 .
\end{array}
\end{aligned}
$$

Moreover, from (5.1), (5.8), (5.10), and (5.13), for arbitrary $x, y \in \mathbb{R}$ and $\omega \in \Omega$ we find that $l_{0}(x)+m_{0}(y)=x y$ if and only if $x=x_{0}$ and that $l_{T}(\omega, x)+m_{T}(\omega, y)=-x y$ if and only if $x=-(y+c(\omega)) / a(\omega)$. From these equivalences and (5.29), (5.3), and (5.5), it follows that, for an arbitrary $(\bar{X}, \bar{Y}) \in \mathbb{B} \times \mathbb{B},(5.25)-(5.28)$ hold if and only if (5.18)-(5.20) hold. However, in view of (5.11) and the universal inequality (5.17), we see that the equality $\Phi(\bar{X})+\Psi(\bar{Y})=0$ is equivalent to (5.24); hence, (5.24) is equivalent to items (5.18)-(5.20). The result follows from this, given the equivalence of (5.25)-(5.28) and (5.18)-(5.20) just noted.

Remark 5.4. It follows from Proposition 5.3 that the solution of problem $\left(\mathcal{P}_{c, q}\right)$ in $(5.2)$ reduces to the construction of a pair $(\bar{X}, \bar{Y}) \in \mathbb{B} \times \mathbb{B}$ which satisfies the optimality relations (5.25)(5.28), since then the optimal portfolio $\overline{\boldsymbol{\pi}}$ is defined by (5.28). Motivated by (5.20), in the remainder of this subsection we show that there exists a solution to the problem of minimizing $\Psi(\cdot)$ on $\mathbb{B}$, henceforth referred to as the dual problem. Define

$$
\mathbb{B}_{1}:=\left\{Y \equiv\left(Y_{0}, \dot{Y}, \boldsymbol{\Lambda}_{Y}\right) \in \mathbb{B}: \dot{Y}(t)=-r(t) Y(t) \text { a.e. }\right\},
$$

and observe from (5.16) and (5.14) that $\Psi$ necessarily takes the value $\infty$ on $\mathbb{B}-\mathbb{B}_{1}$. Then

$$
\inf _{Y \in \mathbb{B}} \Psi(Y)=\inf _{Y \in \mathbb{B}_{1}} \Psi(Y),
$$

and the dual problem reduces to the minimization of $\Psi(\cdot)$ over $\mathbb{B}_{1}$. For each $t \in[0, T]$, let

$$
\begin{gathered}
\beta(t):=\exp \left[-\int_{0}^{t} r(\tau) \mathrm{d} \tau\right], \quad \Im(\boldsymbol{\gamma})(t):=\int_{0}^{t} \beta^{-1}(\tau) \boldsymbol{\gamma}^{\top}(\tau) \mathrm{d} \boldsymbol{W}(\tau), \quad \boldsymbol{\gamma} \in L_{22}, \\
\Xi(y, \boldsymbol{\gamma})(t):=\beta(t)[y+\Im(\boldsymbol{\gamma})(t)], \quad t \in[0, T],(y, \boldsymbol{\gamma}) \in \mathbb{R} \times L_{22} .
\end{gathered}
$$


Remark 5.5. From Section 3 , we see that $Y \equiv\left(Y_{0}, \dot{Y}, \boldsymbol{\Lambda}_{Y}\right) \in \mathbb{B}_{1}$ satisfies the relation

$$
Y(t)=Y_{0}-\int_{0}^{t} r(\tau) Y(\tau) \mathrm{d} \tau+\int_{0}^{t} \boldsymbol{\Lambda}_{Y}^{\top}(\tau) \mathrm{d} \boldsymbol{W}(\tau) .
$$

It follows from Itô's formula and Doob's $L^{2}$-inequality that $\Xi(\cdot, \cdot): \mathbb{R} \times L_{22} \rightarrow \mathbb{B}_{1}$ is a linear bijection, and that if $Y:=\Xi(y, \boldsymbol{\gamma})$ for some $(y, \boldsymbol{\gamma}) \in \mathbb{R} \times L_{22}$ then (recalling (5.23))

$$
Y_{0}=y, \quad \boldsymbol{\Lambda}_{Y}(t)=\boldsymbol{\gamma}(t) \quad \text { a.e., } \quad \boldsymbol{\Theta}_{Y}(t)=-\boldsymbol{\sigma}(t)[\boldsymbol{\theta}(t) Y(t)+\boldsymbol{\gamma}(t)] \quad \text { a.e. }
$$

We then obtain

$$
\begin{aligned}
\inf _{(y, \boldsymbol{\gamma}) \in \mathbb{R} \times L_{22}} \tilde{\Psi}(y, \boldsymbol{\gamma})= & \inf _{Y \in \mathbb{B}_{1}} \Psi(Y), \\
& \text { where } \quad \tilde{\Psi}(y, \boldsymbol{\gamma}):=\Psi(\Xi(y, \boldsymbol{\gamma})), \quad(y, \boldsymbol{\gamma}) \in \mathbb{R} \times L_{22} .
\end{aligned}
$$

Moreover, $M\left(t, Y(t), \dot{Y}(t), \boldsymbol{\Lambda}_{Y}(t)\right)=\delta\left(\boldsymbol{\Theta}_{Y}(t)\right)$ a.e. for each $Y \in \mathbb{B}_{1}$ (see (5.30) and (5.14)) and, thus, from (5.13) and (5.16), for each $(y, \boldsymbol{\gamma}) \in \mathbb{R} \times L_{22}$ (with $Y:=\Xi(y, \boldsymbol{\gamma})$ ) we have

$$
\tilde{\Psi}(y, \boldsymbol{\gamma})=x_{0} y+\mathrm{E}\left[\frac{(Y(T)+c)^{2}}{2 a}\right]+\mathrm{E}\left[\int_{0}^{T} \delta\left(\boldsymbol{\Theta}_{Y}(t)\right) \mathrm{d} t\right]-q .
$$

Remark 5.6. We define the norm $\|\cdot\|_{L_{22}}$ on the real vector space $L_{22}$ by

$$
\|\boldsymbol{\gamma}\|_{L_{22}}^{2}:=\mathrm{E}\left[\int_{0}^{T}\|\boldsymbol{\gamma}(t)\|^{2} \mathrm{~d} t\right]
$$

and the norm $\|(\cdot, \cdot)\|$ on the real vector space $\mathbb{R} \times L_{22}$ by $\|(y, \gamma)\|^{2}:=|y|^{2}+\|\boldsymbol{\gamma}\|_{L_{22}}^{2}$. With this norm, $\mathbb{R} \times L_{22}$ is a reflexive Banach space.

Proposition 5.4. Suppose that Conditions 2.1, 4.1, and 5.1 are satisfied. Then

$$
\inf _{(y, \boldsymbol{\gamma}) \in \mathbb{R} \times L_{22}} \tilde{\Psi}(y, \boldsymbol{\gamma})=\tilde{\Psi}(\bar{y}, \overline{\boldsymbol{\gamma}}) \in \mathbb{R} \quad \text { for some }(\bar{y}, \overline{\boldsymbol{\gamma}}) \in \mathbb{R} \times L_{22} .
$$

Proof. It is immediate from (5.37), (5.35), and (5.15) that $\tilde{\Psi}$ is convex on $\mathbb{R} \times L_{22}$. From Conditions 4.1 and 5.1 we have both $\tilde{\Psi}(y, \boldsymbol{\gamma}) \geq x_{0} y-q>-\infty$, for each $(y, \boldsymbol{\gamma}) \in \mathbb{R} \times L_{22}$, and $\tilde{\Psi}(0, \mathbf{0})=\mathrm{E}\left[c^{2} /(2 a)\right]-q<\infty$; hence, $\tilde{\Psi}$ is proper. A routine argument using Fatou's lemma and the nonnegativity and lower semicontinuity of $\delta(\cdot)$ proves that $\tilde{\Psi}$ is lower semicontinuous on $\mathbb{R} \times L_{22}$ (with respect to the norm $\|(\cdot, \cdot)\|$ ). We next show that $\tilde{\Psi}$ is coercive (that is, $\tilde{\Psi}(y, \boldsymbol{\gamma}) \rightarrow$ $\infty$ as $\|(y, \boldsymbol{\gamma})\| \rightarrow \infty)$. From Conditions 2.1 and 5.1 we know that $\beta^{-1}(T) c$ is $\left\{\mathcal{F}_{T}\right\}$-measurable and square integrable and, thus, can be written $\beta^{-1}(T) c=\tilde{y}+\int_{0}^{T} \eta^{\top}(\tau) \mathrm{d} \boldsymbol{W}(\tau)$ for $\tilde{y}=$ $\mathrm{E}\left[\beta^{-1}(T) c\right]$ and some $\eta \in L_{22}$ (see [8, Theorem 3.4.15]). From (5.32) and (5.33) we therefore obtain $\Xi(y, \boldsymbol{\gamma})(T)+c=\Xi(y+\tilde{y}, \boldsymbol{\gamma}+\beta \boldsymbol{\eta})(T),(y, \boldsymbol{\gamma}) \in \mathbb{R} \times L_{22}$. Thus, to demonstrate coercivity, with no loss of generality we can and shall take $c \equiv 0$ in (5.37). In view of the nonrandom and strictly positive uniform lower bounds on $\beta(T)$ and $1 /(2 a)$ (see Conditions 2.1 and 4.1), and the Itô isometry, we find that $\mathrm{E}\left[\Xi(y, \gamma)^{2}(T) /(2 a)\right] \rightarrow \infty$ as $\|(y, \gamma)\| \rightarrow \infty$. Coercivity of $\tilde{\Psi}$ follows from this, (5.37), and the nonnegativity of $\delta(\cdot)$. The existence of a pair $(\bar{y}, \bar{\gamma}) \in \mathbb{R} \times L_{22}$ which satisfies (5.38) follows in turn from this, Remark 5.6, and [7, Proposition II-1.2].

Remark 5.7. Define $\bar{Y}:=\Xi(\bar{y}, \bar{\gamma})$ for the pair $(\bar{y}, \bar{\gamma}) \in \mathbb{R} \times L_{22}$ given by Proposition 5.4. From Remark 5.5 we have $\bar{Y} \in \mathbb{B}_{1} \subset \mathbb{B}$. Upon combining (5.38), (5.36), and (5.31), we obtain $\Psi(\bar{Y})=\inf _{Y \in \mathbb{B}} \Psi(Y)$; thus, $\bar{Y}$ solves the dual problem of Remark 5.4. 


\subsection{Construction of the optimal portfolio}

In this subsection we shall construct an $\bar{X} \in \mathbb{B}$ such that the pair $(\bar{X}, \bar{Y})$ with $\bar{Y}$ as defined in Remark 5.7 satisfies (5.25)-(5.28). To this end, consider the state price density process given by (recalling $\beta$ from (5.32))

$$
H(t):=\beta(t) \mathcal{E}\left(-\boldsymbol{\theta}^{\top} \bullet \boldsymbol{W}\right)(t)
$$

Remark 5.8. In (5.39) the notation $\&(M)(t):=\exp \left[M(t)-\frac{1}{2}\langle M\rangle(t)\right]$ indicates the exponential of a continuous local martingale $M$ (with $\langle M\rangle$ denoting the quadratic variation of $M$ ), and ' $\bullet$ ' denotes stochastic integration.

Remark 5.9. Fix an arbitrary $p \in \mathbb{R}$. Since $\boldsymbol{\theta}$ is uniformly bounded (recall Remark 2.2), it follows that $\left\{\mathscr{E}\left(-p \boldsymbol{\theta}^{\top} \bullet \boldsymbol{W}\right)(t)\right\}$ is a continuous $\left\{\mathcal{F}_{t}\right\}$-martingale (by the Novikov criterion; see [8, Corollary 3.5.14]). It then easily follows from the uniform boundedness of $\boldsymbol{\theta}$ and $r$ (see Condition 2.1), and Doob's maximal $L^{2}$-inequality, that $\mathrm{E}\left[\sup _{t \in[0, T]}|H(t)|^{p}\right]<\infty$ for each $p \in \mathbb{R}$. Thus, $H$ as defined in (5.39) is a member of $\mathbb{B}$ (set $p=2$ ).

Now, $\left\{\left(H(t) X^{\pi}(t), \mathscr{F}_{t}\right), t \in[0, T]\right\}$ is a martingale for each $\pi \in L_{22}$ (as follows from (5.39), (2.4), Proposition 5.1, Remark 5.9, and Proposition 3.1). This, together with (5.26), motivates the following definition of $\bar{X}$ in terms of $\bar{Y}$ as defined in Remark 5.7:

$$
\bar{X}(t):=-\frac{1}{H(t)} \mathrm{E}\left[\frac{\bar{Y}(T)+c}{a} H(T) \mid \mathcal{F}_{t}\right] .
$$

Remark 5.10. The square integrability of $\bar{Y}(T)$ (recall that $\bar{Y} \in \mathbb{B}$ ) and $c$ (see Condition 5.1), and the strictly positive lower bound on $a$ (see Condition 4.1), ensure that $(\bar{Y}(T)+c) / a$ is square integrable. Together with Remark 5.9, this establishes the existence of the conditional expectation in (5.40).

Observe that $\bar{X}$ as defined in (5.40) satisfies the 'dynamical part' of (2.4), that is

$$
\mathrm{d} \bar{X}(t)=\left\{r(t) \bar{X}(t)+\overline{\boldsymbol{\pi}}^{\top}(t) \boldsymbol{\sigma}(t) \boldsymbol{\theta}(t)\right\} \mathrm{d} t+\overline{\boldsymbol{\pi}}^{\top}(t) \boldsymbol{\sigma}(t) \mathrm{d} \boldsymbol{W}(t),
$$

for some $\mathbb{R}^{N}$-valued $\overline{\boldsymbol{\pi}} \in \mathcal{F}^{*}$ such that $\int_{0}^{T}\|\overline{\boldsymbol{\pi}}(t)\|^{2} \mathrm{~d} t<\infty$ a.s. Indeed, from (5.40) and the martingale representation theorem (see [8, Problem 3.4.16, p. 184]), there exists some $\mathbb{R}^{N}$-valued and a.e. unique $\boldsymbol{\psi} \in \mathcal{F}^{*}$, with $\int_{0}^{T}\|\boldsymbol{\psi}(t)\|^{2} \mathrm{~d} t<\infty$ a.s., such that

$$
\bar{X}(t) H(t)=\bar{X}(0)+\int_{0}^{t} \boldsymbol{\psi}^{\top}(\tau) \mathrm{d} \boldsymbol{W}(\tau):=\xi_{0}(t) .
$$

By expanding the quotient $\bar{X}(t):=\xi_{0}(t) / H(t)$ using Itô's formula, we obtain (5.41) with

$$
\overline{\boldsymbol{\pi}}(t):=\left[\boldsymbol{\sigma}^{\top}\right]^{-1}(t)\left[\frac{\boldsymbol{\psi}(t)}{H(t)}+\bar{X}(t) \boldsymbol{\theta}(t)\right]
$$

(since $\bar{X}$ as defined in (5.40) is continuous, Remark 2.1 shows that $\int_{0}^{T}\|\overline{\boldsymbol{\pi}}(t)\|^{2} \mathrm{~d} t<\infty$ a.s.).

Remark 5.11. From Remark 5.7 we have seen that $\bar{Y} \in \mathbb{B}_{1}$, meaning that (5.27) holds (recall (5.30)), and of course (5.26) is immediate from (5.40). In the remainder of this section we shall establish that $\bar{X} \in \mathbb{B}$ (in which case we see from (5.41) that $\overline{\boldsymbol{\pi}}$ is given by $\overline{\boldsymbol{\pi}}=\left[\boldsymbol{\sigma}^{\top}\right]^{-1} \boldsymbol{\Lambda}_{\bar{X}}$ also), and that (5.25) and (5.28) hold. We shall then have verified (5.25)-(5.28) and will be 
able to conclude that (5.24) holds (from Proposition 5.3), which, together with (5.11), implies that $\Phi(\bar{X})=\vartheta_{c, q}$. Moreover, from (5.28) we obtain $\bar{\pi} \in \mathcal{A}$ (recall (5.3)), while the dynamical relation (5.41), together with (5.25), establishes that $\bar{X}=X^{\bar{\pi}}$ a.e. (for $X^{\pi}$ as defined in the wealth equation (2.4)). However, in light of (5.25), (5.28), (5.8), and (5.7), the first and third terms on the right-hand side of (5.9) equal 0 for $X:=\bar{X}$, implying (from (5.10)) that $\Phi(\bar{X})=\mathrm{E}[J(\bar{X}(T))]$. Thus, $\vartheta_{c, q}=\mathrm{E}\left[J\left(X^{\bar{\pi}}(T)\right)\right]$ and, hence, $\bar{\pi} \in \mathcal{A}$ solves problem $\left(\mathcal{P}_{c, q}\right)$.

Lemma 5.1. Assume that Conditions 2.1, 4.1, and 5.1 are satisfied. Then

$$
\mathrm{E}\left[\sup _{t \in[0, T]}|\bar{X}(t)|^{2}\right]<\infty
$$

(for $\bar{X}$ as defined in (5.40)).

Proof. Let $D:=(\bar{Y}(T)+c) / a$. Then $\mathrm{E}\left[|D|^{2}\right]<\infty$ (see Remark 5.10), and it follows from the integrability of $H(t)$ indicated in Remark 5.9, together with Hölder's inequality, that $D H(T) H^{-1}(t)$ is integrable. Thus, from (5.40) we have $\bar{X}(t)=-\mathrm{E}\left[D H(T) H^{-1}(t) \mid \mathcal{F}_{t}\right]$. Now fix some $q \in(1,2)$ and let $p \in(2, \infty)$ be the conjugate constant given by $p^{-1}+q^{-1}=1$. Hölder's inequality for conditional expectations (see [3, Theorem 7.2.4]) then gives

$$
|\bar{X}(t)| \leq \mathrm{E}\left[\left(\frac{H(T)}{H(t)}\right)^{p} \mid \mathcal{F}_{t}\right]^{1 / p} \mathrm{E}\left[|D|^{q} \mid \mathcal{F}_{t}\right]^{1 / q} \text { a.s. }
$$

for each $t \in[0, T]$. From (5.39) and (5.32), along with the uniform bounds on $r$ and $\boldsymbol{\theta}$ (see Condition 2.1 and Remark 2.2), there exists a constant $k \in(0, \infty)$ such that

$$
\left(\frac{H(T)}{H(t)}\right)^{p} \leq k \frac{\mathcal{E}\left(-p \boldsymbol{\theta}^{\top} \bullet \boldsymbol{W}\right)(T)}{\mathcal{E}\left(-p \boldsymbol{\theta}^{\top} \bullet \boldsymbol{W}\right)(t)} \quad \text { a.s. }
$$

As noted in Remark 5.9, $\left\{\mathcal{E}\left(-p \boldsymbol{\theta}^{\top} \bullet \boldsymbol{W}\right)(t)\right\}$ is an $\left\{\mathcal{F}_{t}\right\}$-martingale; thus, it follows from (5.45) that the first conditional expectation on the right-hand side of (5.44) is a.s. bounded from above by the constant $k$ and, therefore, that

$$
|\bar{X}(t)|^{q} \leq k^{q / p} \mathrm{E}\left[|D|^{q} \mid \mathcal{F}_{t}\right] \quad \text { a.s. }
$$

for each $t \in[0, T]$. Since $\mathrm{E}\left[|D|^{2}\right]<\infty$ and $q \in(1,2)$, we have $\mathrm{E}\left[|D|^{q}\right]<\infty$. Thus, defining $N(t):=\mathrm{E}\left[|D|^{q} \mid \mathcal{F}_{t}\right]$, we find that $\{N(t)\}$ is an $\left\{\mathcal{F}_{t}\right\}$-martingale. Let $p_{1}:=2 / q>1$, where the strict inequality follows since $q \in(1,2)$. From Jensen's inequality we then see that $\mathrm{E}\left[|N(t)|^{p_{1}}\right] \leq \mathrm{E}\left[|D|^{2}\right]<\infty$ for each $t \in[0, T]$ and, consequently, that

$$
\mathrm{E}\left[\sup _{t \in[0, T]}|N(t)|^{p_{1}}\right] \leq\left(\frac{p_{1}}{p_{1}-1}\right)^{p_{1}} \mathrm{E}\left[|N(T)|^{p_{1}}\right]<\infty
$$

(from Doob's $L^{p_{1}}$-inequality). From (5.46) and the definition of $N(t)$ we have $|\bar{X}(t)|^{2} \leq$ $k^{2 / p}|N(t)|^{p_{1}}$, and the result follows from (5.47).

Lemma 5.2. Suppose that Conditions 2.1, 4.1, and 5.1 are satisfied. For $\bar{X}$ and $\overline{\boldsymbol{\pi}}$ as respectively defined in (5.40) and (5.43), we have $\bar{X} \in \mathbb{B}$ and $\bar{\pi} \in L_{22}$.

Proof. For each $n=1,2, \ldots$, let

$$
\tau_{n}:=\inf \left\{t \in[0, T]: \int_{0}^{t}\|\overline{\boldsymbol{\pi}}(s)\|^{2} \mathrm{~d} s \geq n\right\} \wedge T .
$$


Then $\tau_{n}$ is an $\left\{\mathcal{F}_{t}\right\}$-stopping time (recall (2.1)) and $\tau_{n} \uparrow T$ a.s. (since $\int_{0}^{T}\|\overline{\boldsymbol{\pi}}(s)\|^{2} \mathrm{~d} s<\infty$ a.s., as noted following (5.43)). We have seen that $\bar{X}$ and $\bar{\pi}$ are related by (5.41); using this relation to expand $t \mapsto \bar{X}^{2}(t)$ using Itô's formula, and evaluating the expansion at $t \wedge \tau_{n}$, we obtain

$$
\begin{aligned}
\bar{X}^{2}\left(t \wedge \tau_{n}\right)= & \bar{X}^{2}(0)+\int_{0}^{t \wedge \tau_{n}}\left\{2 \bar{X}(s)\left[r(s) \bar{X}(s)+\overline{\boldsymbol{\pi}}^{\top}(s) \boldsymbol{\sigma}(s) \boldsymbol{\theta}(s)\right]+\left\|\boldsymbol{\sigma}^{\top}(s) \overline{\boldsymbol{\pi}}(s)\right\|^{2}\right\} \mathrm{d} s \\
& +2 \int_{0}^{t \wedge \tau_{n}} \bar{X}(s) \overline{\boldsymbol{\pi}}^{\top}(s) \boldsymbol{\sigma}(s) \mathrm{d} \boldsymbol{W}(s), \quad t \in[0, T] .
\end{aligned}
$$

It follows from Lemma 5.1 and the definition of $\tau_{n}$ that the last term on the right-hand side of (5.48) defines an $\left\{\mathcal{F}_{t}\right\}$-martingale null at $t=0$ and, hence, has zero expectation for all $t$; thus, upon taking expectations on each side of (5.48) for $t:=T$, and using the nonnegativity of $r$ (see Condition 2.1), we obtain

$$
\mathrm{E}\left[\bar{X}^{2}\left(\tau_{n}\right)\right]+\mathrm{E}\left[\int_{0}^{\tau_{n}}\left\{-2 \bar{X}(s) \overline{\boldsymbol{\pi}}^{\top}(s) \boldsymbol{\sigma}(s) \boldsymbol{\theta}(s)\right\} \mathrm{d} s\right] \geq \mathrm{E}\left[\int_{0}^{\tau_{n}}\left\|\boldsymbol{\sigma}^{\top}(s) \overline{\boldsymbol{\pi}}(s)\right\|^{2} \mathrm{~d} s\right] .
$$

For arbitrary $\boldsymbol{v}_{1}, \boldsymbol{v}_{2} \in \mathbb{R}^{N}$, we have $\boldsymbol{v}_{1}^{\top} \underline{v}_{2} \leq \frac{1}{2}\left[\left\|\boldsymbol{v}_{1}\right\|^{2}+\left\|\boldsymbol{v}_{2}\right\|^{2}\right]$, from which follows the inequality $-2 \bar{X}(s) \boldsymbol{\theta}^{\top}(s) \boldsymbol{\sigma}^{\top}(s) \overline{\boldsymbol{\pi}}(s) \leq \frac{1}{2}\left[4 \bar{X}^{2}(s)\|\boldsymbol{\theta}(s)\|^{2}+\left\|\boldsymbol{\sigma}^{\top}(s) \overline{\boldsymbol{\pi}}(s)\right\|^{2}\right]$. Substituting this inequality into (5.49) and simplifying then gives

$$
\frac{1}{2} \mathrm{E}\left[\int_{0}^{\tau_{n}}\left\|\boldsymbol{\sigma}^{\top}(s) \overline{\boldsymbol{\pi}}(s)\right\|^{2} \mathrm{~d} s\right] \leq\left(1+T k_{1}\right) \mathrm{E}\left[\sup _{t \in[0, T]}|\bar{X}(t)|^{2}\right], \quad n=1,2, \ldots,
$$

for some constant $k_{1} \in[0, \infty)$ depending only on the uniform bound on $\boldsymbol{\theta}$ (see Remark 2.2). Since $\tau_{n} \uparrow T$ a.s., we obtain $\overline{\boldsymbol{\pi}} \in L_{22}$ from Lemma 5.1 and Remark 2.1 upon letting $n \rightarrow \infty$ in (5.50). Finally, from $\overline{\boldsymbol{\pi}} \in L_{22}$, (5.41), and an argument identical to that for Proposition 3.1, we obtain $\bar{X} \in \mathbb{B}$ (this proof was motivated by the argument for establishing the existence of solutions to backward stochastic differential equations; see, for example, [16, p. 352]).

Recalling Remark 5.11, it remains to verify (5.25) and (5.28). To do so we need the following result.

Lemma 5.3. Assume that Conditions 2.1, 4.1, and 5.1 are satisfied. For an arbitrary $(\alpha, \eta) \in$ $\mathbb{R} \times L_{22}$ and $R:=\Xi(\alpha, \eta)($ see $(5.33))$, we have

$$
0 \leq \alpha\left(x_{0}-\bar{X}(0)\right)+\lim _{\varepsilon \searrow 0} \mathrm{E}\left[\int_{0}^{T}\left\{\frac{\delta\left(\boldsymbol{\Theta}_{\bar{Y}}(t)+\varepsilon \boldsymbol{\Theta}_{R}(t)\right)-\delta\left(\boldsymbol{\Theta}_{\bar{Y}}(t)\right)}{\varepsilon}+\overline{\boldsymbol{\pi}}^{\top}(t) \boldsymbol{\Theta}_{R}(t)\right\} \mathrm{d} t\right] .
$$

Remark 5.12. From (5.38), Remark 5.7, and (5.37), we have $\mathrm{E}\left[\delta\left(\boldsymbol{\Theta}_{\bar{Y}}(t)\right) \mathrm{d} t\right]<\infty$. Thus, the expectation in (5.51) exists in $(-\infty, \infty]$. Since $\delta(\cdot)$ is convex, it follows from [7, p. 23] that the limit on the right-hand side of (5.51) exists (in the extended reals).

Proof of Lemma 5.3. For an arbitrary $\varepsilon \in(0, \infty)$, define $\left(y^{\varepsilon}, \boldsymbol{\gamma}^{\varepsilon}\right) \in \mathbb{R} \times L_{22}$ by $y^{\varepsilon}:=$ $\bar{y}+\varepsilon \alpha$ and $\boldsymbol{\gamma}^{\varepsilon}:=\overline{\boldsymbol{\gamma}}+\varepsilon \boldsymbol{\eta}$. From (5.38) we have $\varepsilon^{-1}\left[\tilde{\Psi}\left(y^{\varepsilon}, \boldsymbol{\gamma}^{\varepsilon}\right)-\tilde{\Psi}(\bar{y}, \overline{\boldsymbol{\gamma}})\right] \geq 0$ for each $\varepsilon \in(0, \infty)$. Using (5.26) (which holds in view of (5.40)) and (5.37) to calculate the quantity $\lim _{\varepsilon \rightarrow 0} \varepsilon^{-1}\left[\tilde{\Psi}\left(y^{\varepsilon}, \boldsymbol{\gamma}^{\varepsilon}\right)-\tilde{\Psi}(\bar{y}, \bar{\gamma})\right]$, we easily obtain

$$
0 \leq \alpha x_{0}-\mathrm{E}[\bar{X}(T) R(T)]+\lim _{\varepsilon \searrow 0} \mathrm{E}\left[\int_{0}^{T} \frac{\delta\left(\boldsymbol{\Theta}_{\bar{Y}}(t)+\varepsilon \boldsymbol{\Theta}_{R}(t)\right)-\delta\left(\boldsymbol{\Theta}_{\bar{Y}}(t)\right)}{\varepsilon} \mathrm{d} t\right] .
$$


Now, from (5.41) and the fact that $\bar{X} \in \mathbb{B}$ (see Lemma 5.2), it follows that

$$
\dot{\bar{X}}(t)=r(t) \bar{X}(t)+\overline{\boldsymbol{\pi}}^{\top}(t) \boldsymbol{\sigma}(t) \boldsymbol{\theta}(t) \quad \text { a.e. } \quad \text { and } \quad \boldsymbol{\Lambda}_{\bar{X}}(t)=\boldsymbol{\sigma}^{\top}(t) \overline{\boldsymbol{\pi}}(t) \quad \text { a.e. }
$$

In view of these observations and Remark 5.5 (applied to $R=\Xi(\alpha, \eta)$ ), it follows from Proposition 5.1 that

$$
\mathbb{M}(\bar{X}, R)(t)=\bar{X}(t) R(t)-\alpha \bar{X}(0)+\int_{0}^{t} \overline{\boldsymbol{\pi}}^{\top}(\tau) \boldsymbol{\Theta}_{R}(\tau) \mathrm{d} \tau
$$

is a continuous $\left\{\mathcal{F}_{t}\right\}$-martingale null at the origin, whence

$$
\mathrm{E}[\mathrm{M}(\bar{X}, R)(t)]=\mathrm{E}[\bar{X}(t) R(t)]-\alpha \bar{X}(0)+\mathrm{E}\left[\int_{0}^{t} \overline{\boldsymbol{\pi}}^{\top}(\tau) \boldsymbol{\Theta}_{R}(\tau) \mathrm{d} \tau\right]=0 .
$$

Combining this with (5.52) gives (5.51).

Lemma 5.4. Assume that Condition 2.1 is satisfied. For each $\rho \in L_{22}$, there exists a unique $\boldsymbol{\xi} \in L_{22}$ such that $\rho(t)=\boldsymbol{\xi}(t)+\boldsymbol{\theta}(t) \int_{0}^{t} \boldsymbol{\xi}^{\top}(\tau) \mathrm{d} \boldsymbol{W}(\tau)$ a.e.

The proof of the preceding result is omitted since it is just a simple modification of the usual argument for the existence and uniqueness of solutions to linear integral equations. Using Lemmas 5.3 and 5.4, we can complete the program outlined in Remark 5.11.

Proposition 5.5. Assume that Conditions 2.1, 4.1, and 5.1 are satisfied. Then (5.25) and (5.28) hold for $\bar{Y}$ as defined in Remark 5.7 and $\bar{X}$ as defined in (5.40).

Proof. We first establish (5.25). Fix an arbitrary $\alpha \in \mathbb{R}$. Since $\boldsymbol{\theta} \in L_{22}$ (being uniformly bounded according to Remark 2.2) and $\beta$ is uniformly bounded (see (5.32) and Condition 2.1), upon setting $\rho(t):=-\alpha \boldsymbol{\theta}(t)$ in Lemma 5.4 we see that there is some $\eta \in L_{22}$ such that $-\alpha \boldsymbol{\theta}(t) \beta(t)=\boldsymbol{\eta}(t)+\boldsymbol{\theta}(t) \beta(t) \int_{0}^{t} \beta^{-1}(\tau) \boldsymbol{\eta}^{\top}(\tau) \mathrm{d} \boldsymbol{W}(\tau)$ a.e. From this, together with (5.33) and (5.32), we obtain $\boldsymbol{\eta}(t)+\boldsymbol{\theta}(t) \Xi(\alpha, \boldsymbol{\eta})(t)=\mathbf{0}$ a.e. Upon defining $R:=\Xi(\alpha, \boldsymbol{\eta})$, from Remark 5.5 we find that $\boldsymbol{\Theta}_{R}(t)=\mathbf{0}$ a.e.; thus, Lemma 5.3 gives $0 \leq \alpha\left(x_{0}-\bar{X}(0)\right)$. Equation (5.25) follows since $\alpha \in \mathbb{R}$ is arbitrary.

It remains to establish (5.28). Since (5.41) holds and $\overline{\boldsymbol{\pi}} \in L_{22}$ (see Lemma 5.2), it is enough to show that $\overline{\boldsymbol{\pi}}(t) \in K$ a.e. to conclude that $\overline{\boldsymbol{\pi}} \in \mathcal{U}(\bar{X})$ (recall (5.3) and (4.1)). Since $\delta(\cdot)$ is subadditive and positively homogeneous (see [9, p. 206]), we have $\delta\left(\boldsymbol{\Theta}_{\bar{Y}}(t)+\varepsilon_{\boldsymbol{\Theta}_{R}}(t)\right) \leq$ $\delta\left(\boldsymbol{\Theta}_{\bar{Y}}(t)\right)+\varepsilon \delta\left(\boldsymbol{\Theta}_{R}(t)\right)$ for arbitrary $\varepsilon \in(0, \infty)$ and $R \in \mathbb{B}$. Then, since we have shown that $\bar{X}(0)=x_{0}$, it follows from Lemma 5.3 that, for each $(\alpha, \eta) \in \mathbb{R} \times L_{22}$,

$$
0 \leq \mathrm{E}\left[\int_{0}^{T}\left\{\delta\left(\boldsymbol{\Theta}_{R}(t)\right)+\overline{\boldsymbol{\pi}}^{\top}(t) \boldsymbol{\Theta}_{R}(t)\right\} \mathrm{d} t\right] \text { for } R:=\Xi(\alpha, \boldsymbol{\eta}) .
$$

Let $B:=\{(\omega, t) \in \Omega \times[0, T]: \overline{\boldsymbol{\pi}}(\omega, t) \in K\}$. By [9, Lemma 5.4.2], there exists some $\left\{\mathcal{F}^{*}\right\}$-measurable mapping $\tilde{\boldsymbol{v}}: \Omega \times[0, T] \rightarrow \mathbb{R}^{N}$ such that $\|\tilde{\boldsymbol{v}}(t)\| \leq 1$ and $|\delta(\tilde{\boldsymbol{v}}(t))| \leq 1$ a.e., and

$$
\delta(\tilde{\boldsymbol{v}}(t))+\overline{\boldsymbol{\pi}}^{\top}(t) \tilde{\boldsymbol{v}}(t)=0 \quad \text { a.e. on } B, \quad \delta(\tilde{\boldsymbol{v}}(t))+\overline{\boldsymbol{\pi}}^{\top}(t) \tilde{\boldsymbol{v}}(t)<0 \quad \text { a.e. on } B^{\mathrm{c}} .
$$

Now suppose that $(\mathrm{P} \otimes \lambda)\{(\Omega \times[0, T])-B\}>0$. Then, by (5.54),

$$
0>\mathrm{E}\left[\int_{0}^{T}\left\{\delta(\tilde{\boldsymbol{v}}(t))+\overline{\boldsymbol{\pi}}^{\top}(t) \tilde{\boldsymbol{v}}(t)\right\} \mathrm{d} t\right] .
$$


Let $\rho(t):=-\beta^{-1}(t) \sigma^{-1}(t) \tilde{\boldsymbol{v}}(t)$. Since $\|\tilde{\boldsymbol{v}}(t)\|$ is essentially bounded on $\Omega \times[0, T]$, it follows from the boundedness of $\beta^{-1}$ and $\sigma^{-1}$ (see Remark 2.1) that $\rho \in L_{22}$. Then, from Lemma 5.4, there exists some $\tilde{\boldsymbol{\xi}} \in L_{22}$ such that $-\beta^{-1}(t) \boldsymbol{\sigma}^{-1}(t) \tilde{\boldsymbol{v}}(t)=\tilde{\boldsymbol{\xi}}(t)+\boldsymbol{\theta}(t) \int_{0}^{t} \tilde{\boldsymbol{\xi}}^{\top}(\tau) \mathrm{d} \boldsymbol{W}(\tau)$ a.e. Multiply each side of this equation by $\beta(t) \boldsymbol{\sigma}(t)$ and let $\tilde{\boldsymbol{\eta}}(t):=\beta(t) \tilde{\boldsymbol{\xi}}(t) \in L_{22}$ and $R:=$ $\Xi(0, \tilde{\boldsymbol{\eta}})=\beta(t) \mathfrak{I}(\tilde{\boldsymbol{\eta}})(t)$ (see (5.33)). Then, from (5.32) and Remark 5.5 we obtain $\tilde{\boldsymbol{v}}(t)=$ $-\boldsymbol{\sigma}(t)[\tilde{\boldsymbol{\eta}}(t)+\boldsymbol{\theta}(t) R(t)]=\boldsymbol{\Theta}_{R}(t)$ a.e. From this and (5.55), we obtain

$$
\mathrm{E}\left[\int_{0}^{T}\left\{\delta\left(\boldsymbol{\Theta}_{R}(t)\right)+\overline{\boldsymbol{\pi}}^{\top}(t) \boldsymbol{\Theta}_{R}(t)\right\} \mathrm{d} t\right]<0
$$

(for $R:=\Xi(0, \tilde{\eta})$ ). Since $\tilde{\boldsymbol{\eta}} \in L_{22}$, this inequality contradicts (5.53) and, so,

$$
(\mathrm{P} \otimes \lambda)\{(\Omega \times[0, T])-B\}=0,
$$

that is, $\overline{\boldsymbol{\pi}}(t) \in K$ a.e., as required to establish that $\overline{\boldsymbol{\pi}} \in \mathcal{U}(\bar{X})$.

We next show that

$$
\delta\left(\boldsymbol{\Theta}_{\bar{Y}}(t)\right)+\overline{\boldsymbol{\pi}}^{\top}(t) \boldsymbol{\Theta}_{\bar{Y}}(t)=0 \quad \text { a.e. }
$$

To this end, let $R:=\Xi(-\bar{y},-\bar{\gamma})$. Then, in light of the linearity of $\Xi(\cdot, \cdot)$, and since $\bar{Y}=$ $\Xi(\bar{y}, \overline{\boldsymbol{\gamma}})$ (see Remark 5.7), we have $R=-\bar{Y}$. Thus, $\boldsymbol{\Theta}_{R}=-\boldsymbol{\Theta}_{\bar{Y}}$ (see (5.23)). Since $\delta(\cdot)$ is positively homogeneous, for each $\varepsilon \in(0,1)$ we obtain

$$
\delta\left(\boldsymbol{\Theta}_{\bar{Y}}(t)+\varepsilon \boldsymbol{\Theta}_{R}(t)\right)=\delta\left((1-\varepsilon) \boldsymbol{\Theta}_{\bar{Y}}(t)\right)=(1-\varepsilon) \delta\left(\boldsymbol{\Theta}_{\bar{Y}}(t)\right) \quad \text { a.e. }
$$

From this, together with $\bar{X}(0)=x_{0}$ (which we have already shown) and Lemma 5.3, we obtain the inequality

$$
0 \geq \mathrm{E}\left[\int_{0}^{T}\left\{\delta\left(\boldsymbol{\Theta}_{\bar{Y}}(t)\right)+\overline{\boldsymbol{\pi}}^{\top}(t) \boldsymbol{\Theta}_{\bar{Y}}(t)\right\} \mathrm{d} t\right] .
$$

Now, we have already seen that $\overline{\boldsymbol{\pi}}(t) \in K$ a.e.; thus, $\delta\left(\boldsymbol{\Theta}_{\bar{Y}}(t)\right)+\overline{\boldsymbol{\pi}}^{\top}(t) \boldsymbol{\Theta}_{\bar{Y}}(t) \geq 0$ a.e. (see (5.15)). This, together with the inequality just noted, establishes (5.56). Finally, we see from (5.41) that $\overline{\boldsymbol{\pi}}$ as defined in (5.43) is also given by $\overline{\boldsymbol{\pi}}(t):=\left[\boldsymbol{\sigma}^{\top}\right]^{-1}(t) \boldsymbol{\Lambda}_{\bar{X}}(t)$. This establishes (5.28).

For easy reference, we summarize the main result of the present section as follows.

Proposition 5.6. Suppose that Conditions 2.1, 4.1, and 5.1 are satisfied. Then there exists a pair $(\bar{y}, \bar{\gamma}) \in \mathbb{R} \times L_{22}$ minimizing the proper convex functional $\tilde{\Psi}(\cdot, \cdot)$ (see (5.37)) over $\mathbb{R} \times L_{22}$. Define $\bar{Y}:=\Xi(\bar{y}, \bar{\gamma})$ (with $\Xi$ as given in (5.33) and (5.32)) and $H$ as in (5.39), and let

$$
\bar{X}(t):=-\frac{1}{H(t)} \mathrm{E}\left[\frac{\bar{Y}(T)+c}{a} H(T) \mid \mathcal{F}_{t}\right], \quad \overline{\boldsymbol{\pi}}(t):=\left[\boldsymbol{\sigma}^{\top}\right]^{-1}(t)\left[\frac{\psi(t)}{H(t)}+\bar{X}(t) \boldsymbol{\theta}(t)\right] .
$$

(Here $\psi \in \mathcal{F}^{*}$ is the $\mathbb{R}^{N}$-valued, a.e. unique process on $\Omega \times[0, T]$ such that $\int_{0}^{T}\|\psi(t)\|^{2} \mathrm{~d} t<$ $\infty$ a.s., and $\bar{X}(t) H(t)=\bar{X}(0)+\int_{0}^{t} \boldsymbol{\psi}^{\top}(\tau) \mathrm{d} \boldsymbol{W}(\tau)$, given by the martingale representation theorem.) Then $\overline{\boldsymbol{\pi}} \in \mathcal{A}$ and $\bar{X}(t)=X^{\bar{\pi}}(t)$ a.e. (for $X^{\pi}$ as defined in (2.5)), and

$$
\inf _{\pi \in \mathcal{A}} \mathrm{E}\left[J\left(X^{\pi}(T)\right)\right]=\mathrm{E}\left[J\left(X^{\bar{\pi}}(T)\right)\right]=-\inf _{(y, \gamma) \in \mathbb{R} \times L_{22}} \tilde{\Psi}(y, \boldsymbol{\gamma})=-\tilde{\Psi}(\bar{y}, \bar{\gamma}) \in \mathbb{R} .
$$

In particular, $\overline{\boldsymbol{\pi}}$ solves the partially constrained problem $\left(\mathcal{P}_{c, q}\right)$, given in (5.2). 


\section{The fully constrained optimization problem}

In this section we return to the main goal of the paper, namely the solution of the fully constrained problem (4.5). Our approach relies on Proposition 5.6, together with results from Lagrange duality for convex optimization as set forth in [1, Chapter 2, Section 6].

Throughout this section we postulate Conditions 2.1, 4.1, 4.2, and 4.3. Then

(i) $\mathcal{A}$ is a convex subset of $L_{22}$ (as follows from the convexity of $K$ in Condition 4.1),

(ii) $G$ is an affine functional on $L_{22}$ (as follows from (2.5) and (4.3)), and

(iii) $\pi \mapsto \mathrm{E}\left[\hat{J}\left(X^{\pi}(T)\right)\right]$ defines an $\mathbb{R}$-valued convex mapping on $\mathcal{A}$ (as follows from Proposition 3.1 and Conditions 4.1 and 4.2).

We now define the Lagrangian function for the optimization problem $(\hat{\mathcal{P}})(\operatorname{see}(4.5))$, as follows:

$$
\mathcal{L}(\mu ; \pi):=\mathrm{E}\left[\hat{J}\left(X^{\pi}(T)\right)\right]+\mu G(\pi), \quad \pi \in L_{22}, \mu \in \mathbb{R} .
$$

From [1, Proposition 2.6.1], Condition 4.3, and [1, Theorem 2.6.1], there exists some 'Lagrange multiplier' $\bar{\mu} \in \mathbb{R}$ such that (recalling (4.4))

$$
\hat{\vartheta}=\sup _{\mu \in \mathbb{R}} \inf _{\boldsymbol{\pi} \in \mathcal{A}} \mathcal{L}(\mu ; \boldsymbol{\pi})=\inf _{\boldsymbol{\pi} \in \mathcal{A}} \mathcal{L}(\bar{\mu} ; \boldsymbol{\pi})
$$

For each $(\mu, \omega, x) \in \mathbb{R} \times \Omega \times \mathbb{R}$, let

$$
J_{1}(\mu ; \omega, x):=\frac{1}{2}\left[a(\omega) x^{2}+2 c_{\mu}(\omega) x\right]-\mu d \quad \text { with } \quad c_{\mu}(\omega):=c_{0}(\omega)+\mu c_{1}(\omega),
$$

where $a, c_{0}$, and $c_{1}$ are as given in Conditions 4.1 and 4.2, and observe, from (4.2), (4.3), and (6.1), that

$$
\mathcal{L}(\mu ; \pi)=\mathrm{E}\left[J_{1}\left(\mu ; X^{\pi}(T)\right)\right], \quad \pi \in L_{22}, \mu \in \mathbb{R} .
$$

Remark 6.1. For each fixed $\mu \in \mathbb{R}$, the function $J_{1}(\mu ; \cdot, \cdot)$ is identical to the function $J(\cdot, \cdot)$ in (5.1) with $c_{\mu}$ in place of $c$ and $-\mu d$ in place of $q$. In view of Condition 4.2, we see that $\mathrm{E}\left[c_{\mu}^{2}\right]<\infty$, that is, Condition 5.1 holds with $c_{\mu}$ in place of $c$ for each $\mu \in \mathbb{R}$, and the infima in (6.2) therefore correspond to optimization problems $\left(\mathcal{P}_{c, q}\right)$ of the form (5.2) (with $c:=c_{\mu}$ and $q:=-\mu d$ ), which are addressed in Proposition 5.6.

Motivated by (5.37) and Remark 6.1, for each $(\mu, y, \boldsymbol{\gamma}) \in \mathbb{R} \times \mathbb{R} \times L_{22}$ and $Y:=\Xi(y, \boldsymbol{\gamma})$ we let

$$
\tilde{\Psi}_{1}(\mu ; y, \boldsymbol{\gamma}):=x_{0} y+\mathrm{E}\left[\frac{\left(Y(T)+c_{\mu}\right)^{2}}{2 a}\right]+\mathrm{E}\left[\int_{0}^{T} \delta\left(\boldsymbol{\Theta}_{Y}(t)\right) \mathrm{d} t\right]+\mu d .
$$

Remark 6.2. Proposition 5.6 asserts the existence of a minimizer, $(\bar{y}(\mu), \overline{\boldsymbol{\gamma}}(\mu)) \in \mathbb{R} \times L_{22}$, of $\tilde{\Psi}_{1}(\mu ; \cdot, \cdot)$ over $\mathbb{R} \times L_{22}$ for each $\mu \in \mathbb{R}$; motivated by Proposition 5.6 , we define

$$
\begin{aligned}
& \bar{Y}(\mu ; t):=\Xi(\bar{y}(\mu), \bar{\gamma}(\mu))(t), \\
& \bar{X}(\mu ; t):=-\frac{1}{H(t)} \mathrm{E}\left[\frac{\bar{Y}(\mu ; T)+c_{\mu}}{a} H(T) \mid \mathcal{F}_{t}\right], \\
& \overline{\boldsymbol{\pi}}(\mu ; t):=\left[\boldsymbol{\sigma}^{\top}\right]^{-1}(t)\left[\frac{\psi(\mu ; t)}{H(t)}+\bar{X}(\mu ; t) \boldsymbol{\theta}(t)\right],
\end{aligned}
$$


for each $\mu \in \mathbb{R}$. From (6.7) and the martingale representation theorem, there exists some a.e. unique, $\mathbb{R}^{N}$-valued, $\left\{\mathcal{F}_{t}\right\}$-progressively measurable process $\psi(\mu ; \cdot)$ on $\Omega \times[0, T]$ such that $\int_{0}^{T}\|\boldsymbol{\psi}(\mu ; t)\|^{2} \mathrm{~d} t<\infty$ a.s. and $\bar{X}(\mu ; t) H(t)=\bar{X}(\mu ; 0)+\int_{0}^{t} \boldsymbol{\psi}^{\top}(\mu ; \tau) \mathrm{d} \boldsymbol{W}(\tau)$ for all $t \in[0, T]$; it is this process which appears on the right-hand side of (6.8). Finally, note from Proposition 5.6 that $\bar{\pi}(\mu) \in \mathcal{A}$ and $\bar{X}(\mu ; t)=X^{\bar{\pi}(\mu)}(t)$ a.e. for each $\mu \in \mathbb{R}$ (with $X^{\bar{\pi}(\mu)}$ as given in (2.5)).

It remains to show that $\overline{\boldsymbol{\pi}}(\bar{\mu} ; \cdot)$ solves problem $(\hat{\mathcal{P}})$. From Proposition 5.6 and $(6.4)$, for each $\mu \in \mathbb{R}$ we have

$$
\inf _{\boldsymbol{\pi} \in \mathcal{A}} \mathcal{L}(\mu ; \boldsymbol{\pi})=\mathcal{L}(\mu, \overline{\boldsymbol{\pi}}(\mu))=-\inf _{(y, \boldsymbol{\gamma}) \in \mathbb{R} \times L_{22}} \tilde{\Psi}_{1}(\mu ; y, \boldsymbol{\gamma})=-\tilde{\Psi}_{1}(\mu ; \bar{y}(\mu), \overline{\boldsymbol{\gamma}}(\mu)) \in \mathbb{R}
$$

Since $\overline{\boldsymbol{\pi}}(\bar{\mu}) \in \mathcal{A}$ (by Remark 6.2), it is enough to show that

$$
G(\overline{\boldsymbol{\pi}}(\bar{\mu}))=0,
$$

because then [1, Proposition 2] together with the second equality in (6.2) and the first equality in $(6.9)$ establish that $\overline{\boldsymbol{\pi}}(\bar{\mu})$ solves $(\hat{\mathcal{P}})$. To prove $(6.10)$, we use variational analysis on the optimality of $\bar{\mu}$; from (6.2) and (6.9) we find that

$$
-\hat{\vartheta}=\inf _{(\mu, y, \boldsymbol{\gamma}) \in \mathbb{R} \times \mathbb{R} \times L_{22}} \tilde{\Psi}_{1}(\mu ; y, \boldsymbol{\gamma})=\tilde{\Psi}_{1}(\bar{\mu} ; \bar{y}(\bar{\mu}), \bar{\gamma}(\bar{\mu})) .
$$

Now let $\mu^{\varepsilon}:=\bar{\mu}+\varepsilon \rho$ for $\rho \in \mathbb{R}$ and $\varepsilon \in(0, \infty)$. Then, from (6.11), we have

$$
0 \leq \frac{\tilde{\Psi}_{1}\left(\mu^{\varepsilon} ; \bar{y}(\bar{\mu}), \bar{\gamma}(\bar{\mu})\right)-\tilde{\Psi}_{1}(\bar{\mu} ; \bar{y}(\bar{\mu}), \bar{\gamma}(\bar{\mu}))}{\varepsilon}, \quad \varepsilon \in(0, \infty) .
$$

From the definition of $c_{\mu}$ in (6.3), we have $c_{\mu^{\varepsilon}}=c_{\bar{\mu}}+\varepsilon \rho c_{1}$; hence, from (6.12) and (6.5) we obtain $0 \leq \rho \mathrm{E}\left[\left\{\left(\bar{Y}(\bar{\mu} ; T)+c_{\bar{\mu}}\right) c_{1}\right\} / a\right]+\varepsilon \rho^{2} \mathrm{E}\left[c_{1}^{2} /(2 a)\right]+\rho d$ for all $\varepsilon \in(0, \infty)$. Letting $\varepsilon \rightarrow 0$ and using the arbitrary choice of $\rho \in \mathbb{R}$ then gives $\mathrm{E}\left[\left\{\left(\bar{Y}(\bar{\mu} ; T)+c_{\bar{\mu}}\right) c_{1}\right\} / a\right]+d=0$, which, in view of (6.7), establishes that $\mathrm{E}\left[c_{1} \bar{X}(\bar{\mu} ; T)\right]=d$. Equation (6.10) follows from this together with $\bar{X}(\bar{\mu} ; T)=X^{\bar{\pi}(\bar{\mu})}(T)$ (recall Remark 6.2) and (4.3).

Remark 6.3. We now assemble the preceding results and state the main result of this section. Define $h(\mu):=\inf _{(y, \gamma) \in \mathbb{R} \times L_{22}} \tilde{\Psi}_{1}(\mu ; y, \boldsymbol{\gamma}), \mu \in \mathbb{R}$, and note, from (6.9), that the second equality of (6.2) gives $\inf _{\mu \in \mathbb{R}} h(\mu)=h(\bar{\mu})$.

Proposition 6.1. Suppose that Conditions 2.1, 4.1, 4.2, and 4.3 are satisfied. For each $\mu \in \mathbb{R}$, there exists a pair $(\bar{y}(\mu), \bar{\gamma}(\mu)) \in \mathbb{R} \times L_{22}$ which minimizes the functional $\tilde{\Psi}_{1}(\mu ; \cdot, \cdot)$ over $\mathbb{R} \times L_{22}$ (recall (6.5)) and hence satisfies $h(\mu)=\tilde{\Psi}_{1}(\mu ; \bar{y}(\mu), \bar{\gamma}(\mu))$. Moreover, there exists some $\bar{\mu} \in \mathbb{R}$ which minimizes $h(\cdot)$ on $\mathbb{R}$, and $\hat{\boldsymbol{\pi}}:=\overline{\boldsymbol{\pi}}(\bar{\mu})$ (as given in Remark 6.2 with $\mu:=\bar{\mu}$ ) is the optimal portfolio for the problem $(\hat{\mathcal{P}})$ (see $(4.5))$.

Example 6.1. Take $K:=\mathbb{R}^{N}$ in Condition 4.1 for the unconstrained case. From (5.15) we see that $\delta(\mathbf{0})=0$ and that $\delta(\boldsymbol{z})=\infty$ for $\boldsymbol{z} \neq \mathbf{0}$. Thus, we need minimize $\tilde{\Psi}_{1}(\mu ; \cdot, \cdot)$ only over pairs $(y, \boldsymbol{\gamma}) \in \mathbb{R} \times L_{22}$ such that $\boldsymbol{\Theta}_{Y}(t)=0$ a.e. (for $Y:=\boldsymbol{\Xi}(y, \boldsymbol{\gamma})$ ). From Remark 5.5 and the nonsingularity of $\boldsymbol{\sigma}(t)$ (see Condition 2.1), we obtain $Y_{0}=y$ and $\boldsymbol{\Lambda}_{Y}(t)=-Y(t) \boldsymbol{\theta}(t)$ a.e. Inserting these into (5.34) then shows that $Y(t)=y H(t)$ a.e. and $\gamma \in L_{22}$ necessarily has the form $\boldsymbol{\gamma}(t)=-y H(t) \boldsymbol{\theta}(t)$ a.e. for some $y \in \mathbb{R}$ (recall (5.39)). Determination of the optimal portfolio reduces to the following: (i) for each $\mu \in \mathbb{R}$, locate the minimizer, $\bar{y}(\mu) \in \mathbb{R}$, of 
the functional $y \mapsto \tilde{\Psi}_{2}(\mu ; y):=\tilde{\Psi}_{1}(\mu ; y,-y H \boldsymbol{\theta})$ (which is quadratic); and (ii) use $\bar{y}(\mu)$ to minimize the functional $h(\mu):=\tilde{\Psi}_{2}(\mu ; \bar{y}(\mu)), \mu \in \mathbb{R}$ (which is also quadratic). In the special case of the mean-variance problem of Remark 4.1, where $a=2, c_{0}=0$, and $c_{1}=1$, we have $c_{\mu}=\mu$ (by (6.3)), and (i) and (ii) lead to the (unique) minimizers

$$
\bar{y}(\mu)=-\frac{2 x_{0}+\mu \mathrm{E}[H(T)]}{\mathrm{E}\left[H^{2}(T)\right]} \quad \text { and } \quad \bar{\mu}=2 \frac{x_{0} \mathrm{E}[H(T)]-d \mathrm{E}\left[H^{2}(T)\right]}{\operatorname{var}(H(T))} .
$$

Then $\bar{Y}(\bar{\mu} ; t)=\bar{y}(\bar{\mu}) H(t)$ and $\bar{X}(\bar{\mu} ; T)=-\frac{1}{2}[\bar{Y}(\bar{\mu} ; T)+\bar{\mu}]$ (by (6.7)), the optimal portfolio $\overline{\boldsymbol{\pi}}(\bar{\mu})$ is given by (6.8) with $\mu:=\bar{\mu}$ (by Proposition 6.1), and the least variance (or efficient frontier) is given by

$$
\inf _{\substack{\pi \in L_{22} \\ \mathrm{E}\left[X^{\pi}(T)\right]=d}} \operatorname{var}\left(X^{\pi}(T)\right)=\operatorname{var}(\bar{X}(\bar{\mu} ; T))=\frac{1}{4} \operatorname{var}(\bar{Y}(\bar{\mu} ; T))=\frac{\left(x_{0}-d \mathrm{E}[H(T)]\right)^{2}}{\operatorname{var}(H(T))} .
$$

Example 6.2. We suppose that $K \subset \mathbb{R}^{N}$ in Condition 4.1 is a closed, convex cone, that the market coefficients $r, \boldsymbol{b}$, and $\boldsymbol{\sigma}$ in Condition 2.1 are nonrandom continuous functions on $[0, T]$, and that $c_{0}, c_{1}$, and $a$ in Conditions 4.1 and 4.2 are also nonrandom. In this case the dual problem of minimizing $\tilde{\Psi}_{1}(\mu ; y, \boldsymbol{\gamma})$ over the pairs $(y, \boldsymbol{\gamma}) \in \mathbb{R} \times L_{22}$ (recall (6.5)) is particularly well suited to the application of dynamic programming and leads to an essentially explicit formula for the optimal portfolio $\overline{\boldsymbol{\pi}}(\mu)$ in (6.8). Since $K$ is a convex cone, from (5.15) we have $\delta \equiv 0$ on $\tilde{K}:=\{z: \delta(z)<\infty\}$ (the 'barrier cone' of $-K$ ). Thus, the third term on the right-hand side of (6.5) takes values in the two-point set $\{0, \infty\}$ according to whether or $\operatorname{not} \boldsymbol{\Theta}_{Y}(t) \in \tilde{K}$ a.e. We can therefore regard $\boldsymbol{u}(t):=\boldsymbol{\Theta}_{Y}(t)$ (rather than $\boldsymbol{\gamma}$ ) as the 'control' in the dual problem, and it then follows from (5.34) and (5.35) that the dual process $Y$ satisfies

$$
\mathrm{d} Y(t)=-r(t) Y(t) \mathrm{d} t-\left[\boldsymbol{\theta}(t) Y(t)+\boldsymbol{\sigma}^{-1}(t) \boldsymbol{u}(t)\right]^{\top} \mathrm{d} \boldsymbol{W}(t)
$$

with $\boldsymbol{u}(t) \in \tilde{K}$ a.e.

For each $(y, \boldsymbol{u}) \in \mathbb{R} \times L_{22}$, let $\{\tilde{\Xi}(y, \boldsymbol{u})(t), t \in[0, T]\}$ denote the process $Y$ given in (6.13) with the initial condition $Y(0)=y$. Then, for an arbitrary $\mu \in \mathbb{R}$, the dual problem of mininimizing $\tilde{\Psi}_{1}(\mu ; y, \boldsymbol{\gamma})$ in $(6.5)$ over pairs $(y, \boldsymbol{\gamma}) \in \mathbb{R} \times L_{22}$ is equivalent to the minimization of

$$
\tilde{\Psi}_{3}(\mu ; y, \boldsymbol{u}):=x_{0} y+\mathrm{E}\left[\frac{\left(\tilde{\Xi}(y, \boldsymbol{u})(T)+c_{\mu}\right)^{2}}{2 a}\right]+\mu d
$$

over $(y, \boldsymbol{u}) \in \mathbb{R} \times L_{22}$ with $\boldsymbol{u}(t) \in \tilde{K}$ a.e. (a straightforward application of Gronwall's inequality yields $\tilde{\Xi}(y, \boldsymbol{u}) \in \mathbb{B}$ for each $\left.(y, \boldsymbol{u}) \in \mathbb{R} \times L_{22}\right)$. We now minimize the second term of (6.14) over $\boldsymbol{u} \in L_{22}$ for arbitrary $y \in \mathbb{R}$. Keeping $\mu \in \mathbb{R}$ fixed, define the value function

$$
V(\mu ; y):=\inf _{\substack{\boldsymbol{u} \in L_{22} \\ \boldsymbol{u}(t) \in \tilde{K} \text { a.e. }}} \mathrm{E}\left[\frac{\left(\tilde{\boldsymbol{\Xi}}(y, \boldsymbol{u})(T)+c_{\mu}\right)^{2}}{2 a}\right], \quad y \in \mathbb{R},
$$

and consider the Bellman equation associated with (6.13) and (6.15), namely

$$
\begin{gathered}
\tilde{v}_{s}(s, y)-r(s) y \tilde{v}_{y}(s, y)+\frac{1}{2} \inf _{\eta \in \tilde{K}}\left\{\left\|\boldsymbol{\sigma}^{-1}(s) \boldsymbol{\eta}+\boldsymbol{\theta}(s) y\right\|^{2} \tilde{v}_{y y}(s, y)\right\}=0, \\
\tilde{v}(T, y)=\frac{\left(y+c_{\mu}\right)^{2}}{2 a},
\end{gathered}
$$


for each $(s, y) \in[0, T] \times \mathbb{R}$. This is a particularly tractable equation because the infimum in (6.16) is easily expressible in terms of $y \in \mathbb{R}$. Indeed, for $s \in[0, T]$ and $i=1,2$, let

$$
\zeta_{i}(s):=\underset{\boldsymbol{\eta} \in \tilde{K}}{\arg \min }\left\|\boldsymbol{\sigma}^{-1}(s) \boldsymbol{\eta}-(-1)^{i} \boldsymbol{\theta}(s)\right\|^{2}=\boldsymbol{\sigma}(s) \operatorname{proj}\left((-1)^{i} \boldsymbol{\theta}(s) \mid \boldsymbol{\sigma}^{-1}(s) \tilde{K}\right),
$$

where $\operatorname{proj}(z \mid C)$ is the (uniquely determined) projection of a vector $z \in \mathbb{R}^{N}$ on a closed convex set $C \subset \mathbb{R}^{N}$. Then, for each $s \in[0, T]$, it follows that

$$
\hat{\boldsymbol{u}}(s, y):=\underset{\boldsymbol{\eta} \in \tilde{K}}{\arg \min }\left\|\boldsymbol{\sigma}^{-1}(s) \boldsymbol{\eta}+\boldsymbol{\theta}(s) y\right\|^{2}= \begin{cases}y \zeta_{1}(s) & \text { if } y \geq 0, \\ -y \zeta_{2}(s) & \text { if } y<0 .\end{cases}
$$

In light of (6.19), we can easily write down an explicit solution to (6.16) and (6.17). To this end, for $(s, y) \in[0, T] \times \mathbb{R}$ and $i=1,2$ (recalling $\beta$ from (5.32) and $c_{\mu}$ from (6.3)), define

$$
\begin{gathered}
A_{i}(s):=\exp \left[\int_{s}^{T}\left\|\boldsymbol{\theta}(\tau)-(-1)^{i} \sigma^{-1}(\tau) \zeta_{i}(\tau)\right\|^{2} \mathrm{~d} \tau\right], \\
P_{i}(s):=\frac{1}{a}\left(\frac{\beta(T)}{\beta(s)}\right)^{2} A_{i}(s), \quad \chi(\mu ; s):=\frac{c_{\mu}}{a} \frac{\beta(T)}{\beta(s)}, \quad \alpha(\mu):=\frac{c_{\mu}^{2}}{2 a}, \\
\tilde{v}(\mu ; s, y):=\left\{\begin{array}{l}
P_{1}(s) \frac{y^{2}}{2}+\chi(\mu ; s) y+\alpha(\mu) \quad \text { if }(s, y) \in[0, T] \times[0, \infty), \\
P_{2}(s) \frac{y^{2}}{2}+\chi(\mu ; s) y+\alpha(\mu) \quad \text { if }(s, y) \in[0, T] \times(-\infty, 0) .
\end{array}\right.
\end{gathered}
$$

Then $\tilde{v}(\mu ; \cdot, \cdot)$ is of class $C^{1,1}$ over $[0, T] \times \mathbb{R}$ and of class $C^{1,2}$ over $[0, T] \times(\mathbb{R} \backslash\{0\})$, and a simple, direct verification establishes that it satisfies the Bellman equation (6.16) in the classical sense for each $(s, y) \in[0, T] \times(\mathbb{R} \backslash\{0\})$, as well as the boundary condition (6.17). Moreover, the second-order parabolic sub- and superdifferentials of $\tilde{v}(\mu ; s, y)$ at $(s, y) \in[0, T] \times\{0\}$ are easily computed to show that $\tilde{v}(\mu ; \cdot, \cdot)$ defines a viscosity solution to $(6.16)$ and $(6.17)$ on $[0, T] \times \mathbb{R}$. It now follows from the verification theorem for dynamic programming [16, Theorem 5.3] that $\hat{\boldsymbol{u}}$ in (6.19) is the optimal feedback control for the problem (6.15) with arbitrary $y \in \mathbb{R}$, and $V(\mu ; y)=\tilde{v}(\mu ; 0, y)$ for all $y \in \mathbb{R}$. In particular, the function $y \mapsto V(\mu ; y)=\tilde{v}(\mu ; 0, y)$ is the 'asymmetric quadratic' given in (6.21). Substituting $\hat{\boldsymbol{u}}(t, Y(t))$ for $\boldsymbol{u}(t)$ in $(6.13)$, it follows that the resulting stochastic differential equation has pathwise uniqueness (since $\hat{\boldsymbol{u}}(t, \cdot)$ as given in (6.19) is globally Lipschitz continuous on $\mathbb{R}$ ) and solution (for the initial condition $Y(0)=y \in \mathbb{R})$

$$
\hat{Y}(y ; t):= \begin{cases}y \beta(t) \mathscr{E}\left(-\left[\boldsymbol{\theta}+\boldsymbol{\sigma}^{-1} \zeta_{1}\right]^{\top} \bullet \boldsymbol{W}\right)(t) & \text { if } y \geq 0 \\ y \beta(t) \mathscr{E}\left(-\left[\boldsymbol{\theta}-\boldsymbol{\sigma}^{-1} \zeta_{2}\right]^{\top} \bullet \boldsymbol{W}\right)(t) & \text { if } y<0 .\end{cases}
$$

(recall Remark 5.8). We are now able to minimize $\tilde{\Psi}_{3}(\mu ; \cdot, \cdot)$ in (6.14) (still keeping $\mu \in \mathbb{R}$ fixed). Let $\bar{y}(\mu) \in \mathbb{R}$ be the (unique) minimizer (with respect to $y \in \mathbb{R}$ ) of the 'asymmetric quadratic'

$$
\tilde{\Psi}_{4}(\mu ; y):=x_{0} y+V(\mu ; y)+\mu d=x_{0} y+\tilde{v}(\mu ; 0, y)+\mu d, \quad y \in \mathbb{R},
$$

given by (6.21), and let $\overline{\boldsymbol{u}}(\mu ; t):=\hat{\boldsymbol{u}}(t, \hat{Y}(\bar{y}(\mu) ; t)), t \in[0, T]$. Then $\overline{\boldsymbol{u}}(\mu ; t) \in \tilde{K}$ a.e. (see (6.19)) and the pair $(\bar{y}(\mu), \overline{\boldsymbol{u}}(\mu)) \in \mathbb{R} \times L_{22}$ is the minimizer of the dual cost functional 
$\tilde{\Psi}_{3}(\mu ; \cdot, \cdot)$ defined in (6.14). Comparison of (6.13) with relations (5.34) and (5.35) then shows that, for $\overline{\boldsymbol{\gamma}}(\mu) \in L_{22}$ defined by $\overline{\boldsymbol{\gamma}}(\mu ; t):=-\left[\boldsymbol{\theta}(t) \tilde{\boldsymbol{\Xi}}(\bar{y}(\mu), \overline{\boldsymbol{u}}(\mu))(t)+\boldsymbol{\sigma}^{-1}(t) \overline{\boldsymbol{u}}(\mu ; t)\right]$, the pair $(\bar{y}(\mu), \bar{\gamma}(\mu))$ minimizes the functional $\tilde{\Psi}_{1}(\mu ; \cdot, \cdot)$ (see (6.5)) over $\mathbb{R} \times L_{22}$, and (see (6.6)-(6.8)) the corresponding optimal dual process, $\bar{Y}(\mu)$, is given by $\bar{Y}(\mu ; t)=\hat{Y}(\bar{y}(\mu) ; t), t \in[0, T]$. Using this representation for $\bar{Y}(\mu)$ it is easy to obtain explicit formulae for the portfolio $\overline{\boldsymbol{\pi}}(\mu)$ and the corresponding wealth, $\bar{X}(\mu)$ (see (6.7) and (6.8)). Indeed, upon substituting $\hat{Y}(\bar{y}(\mu) ; T)$ (given in (6.22)) for $\bar{Y}(\mu ; T)$ in (6.7), and using (5.39), the fact that the coefficients $r, \boldsymbol{b}$, and $\boldsymbol{\sigma}$ are deterministic, and the independent increments of $\boldsymbol{W}$, we obtain

$$
-\bar{X}(\mu ; t)=\frac{\hat{Y}(\bar{y}(\mu) ; t)}{a} \exp \left[\int_{t}^{T}\left\{-2 r(\tau)+\boldsymbol{\theta}^{\top}(\tau)\left[\boldsymbol{\theta}(\tau)+\boldsymbol{\sigma}^{-1}(\tau) \zeta_{1}(\tau)\right]\right\} \mathrm{d} \tau\right]+\frac{c_{\mu} \beta(T)}{a \beta(t)}
$$

for $\bar{y}(\mu) \geq 0$ (just replace $\zeta_{1}$ by $-\zeta_{2}$ to obtain $\bar{X}(\mu)$ for $\bar{y}(\mu)<0$ ). Finally, using $(6.22)$ and Itô's product formula to expand the right-hand side of (6.24) (and its analogue for $\bar{y}(\mu)<0$ ), and comparing the result with (2.4), we obtain $\overline{\boldsymbol{\pi}}(\mu)$ such that $\bar{X}(\mu)=X^{\bar{\pi}(\mu)}$ as the following feedback policy on the wealth $\bar{X}(\mu)$ :

$$
\overline{\boldsymbol{\pi}}(\mu ; t):=-\left[\bar{X}(\mu ; t)+a^{-1} \beta^{-1}(t) c_{\mu} \beta(T)\right]\left[\boldsymbol{\sigma}^{\top}\right]^{-1}(t)\left[\boldsymbol{\theta}(t)+\boldsymbol{\sigma}^{-1}(t) \zeta_{1}(t)\right] \text { for } \bar{y}(\mu)>0
$$

$\left(\overline{\boldsymbol{\pi}}(\mu, t)\right.$ is given by (6.25) with $-\zeta_{2}(t)$ in place of $\zeta_{1}(t)$ for $\bar{y}(\mu)<0$, and $\overline{\boldsymbol{\pi}}(\mu, t)=\mathbf{0}$ for $\bar{y}(\mu)=0)$.

We now determine the optimal portfolio and minimum variance in the special case of Remark 4.1, for which $a=2, c_{0}=0$, and $c_{1}=1$. To this end we first characterize the set $\mathcal{R}$ in Remark 4.2. Define the set

$$
F:=\left\{t \in[0, T]:\left\|\boldsymbol{\theta}(t)+\boldsymbol{\sigma}^{-1}(t) \zeta_{1}(t)\right\|>0\right\}=\{t \in[0, T]:-\boldsymbol{\sigma}(t) \boldsymbol{\theta}(t) \notin \tilde{K}\},
$$

where the equality follows from (6.18). Since $\tilde{K}=\left\{z \in \mathbb{R}^{N}:-\pi^{\top} z \leq 0\right.$ for all $\left.\pi \in K\right\}$, we then have $F=\{t \in[0, T]: \Gamma(t) \neq \varnothing\}$, where $\Gamma(t):=\left\{\boldsymbol{\pi} \in K: \boldsymbol{\pi}^{\top} \boldsymbol{\sigma}(t) \boldsymbol{\theta}(t)>0\right\}$. Now suppose that $A_{1}(0)>1$; then $\lambda(F)>0$ (by (6.20)), and by the Aumann selection theorem [15, Theorem 2.3.12] there exists a measurable selection $\pi_{1}(\cdot)$ of $\Gamma(\cdot)$ on $F$. Let $\boldsymbol{\pi}_{2}(t):=\mathbf{0}$ for $t \notin F$ and $\boldsymbol{\pi}_{2}(t):=\boldsymbol{\pi}_{1}(t) /\left\|\boldsymbol{\pi}_{1}(t)\right\|$ for $t \in F$. Then $\boldsymbol{\pi}_{2} \in \mathcal{A}$ (since $K$ is a cone) and $\boldsymbol{\pi}_{2}^{\top}(t) \boldsymbol{\sigma}(t) \boldsymbol{\theta}(t)>0, t \in F$. Hence, from (2.5) we have $\mathrm{E}\left[X^{\pi_{2}}(T)\right]>x_{0} S_{0}(T)$, which establishes that $\left[x_{0} S_{0}(T), \infty\right) \subset \mathcal{R}$ for $A_{1}(0)>1$. Now, if $A_{1}(0)=1$ then $\lambda(F)=0$ (by (6.20)); thus, for each $\pi \in \mathcal{A}$ we have $\pi^{\top}(t) \boldsymbol{\sigma}(t) \boldsymbol{\theta}(t) \leq 0$ a.e. and, hence, $\mathrm{E}\left[X^{\pi}(T)\right] \leq$ $x_{0} S_{0}(T)$ (by (2.5)) and $\mathcal{R} \subset\left(-\infty, x_{0} S_{0}(T)\right]$. In this case the market model is not interesting (in the sense of Remark 4.2), so we shall suppose that $A_{1}(0)>1$ and fix some $d>x_{0} S_{0}(T)$. From Remark 6.3, (6.14), (6.15), and (6.23), we have $h(\mu)=\tilde{\Psi}_{4}(\mu, \bar{y}(\mu)), \mu \in \mathbb{R}$. Using $(6.21)$, it is then easy (although tedious) to calculate that $h(\cdot)$ has the unique minimizer given by

$$
\bar{\mu}=2 \beta^{-1}(T)\left[A_{1}(0)-1\right]^{-1}\left[x_{0}-\beta(T) A_{1}(0) d\right],
$$

and that

$$
\bar{y}(\bar{\mu})=2 \beta^{-2}(T)\left[A_{1}(0)-1\right]^{-1}\left[\beta(T) d-x_{0}\right]>0 .
$$

From this together with (6.22) and the fact that $\operatorname{var}(\bar{X}(\bar{\mu} ; T))=\operatorname{var}(\hat{Y}(\bar{y}(\bar{\mu}) ; T)) / 4($ see $(6.24)$ with $a=2)$, we compute the minimum variance, or efficient frontier, namely

$$
\inf _{\substack{\pi \in \mathcal{A} \\ \mathrm{E}\left[X^{\pi}(T)\right]=d}} \operatorname{var}\left(X^{\pi}(T)\right)=\operatorname{var}(\bar{X}(\bar{\mu} ; T))=\frac{1}{4} \operatorname{var}(\hat{Y}(\bar{y}(\bar{\mu}) ; T))=\frac{\left[x_{0}-\beta(T) d\right]^{2}}{\left[A_{1}(0)-1\right] \beta^{2}(T)} .
$$


The optimal feedback policy is given by (6.25) with $\mu:=\bar{\mu}$ (since we have seen that $\bar{y}(\bar{\mu})>0$ ), and is easy to implement, since only $\zeta_{1}(\cdot)$, given in (6.18), need be 'precalculated off-line' using the known deterministic coefficients $r, \boldsymbol{b}$, and $\boldsymbol{\sigma}$. The simplicity with which dynamic programming applies to the dual problem (for general conical constraints on the portfolio) should be contrasted with the technical complexity involved in applying dynamic programming directly to the primal problem, as in [12], for which the resulting Bellman equation is substantially more involved. As a consequence, the analysis in [12] is very specific to the no-short selling constraint (where $K$ is the positive orthant) and relies on the restriction $b_{n}(t)>r(t), t \in[0, T], n=1, \ldots, N$ (see the text following [12, Equation (2.2)]). This restriction excludes the very natural possibility that interest rates may increase at some point in the investment interval, exceeding - one hopes only temporarily - the mean rates of return on some stocks, and also excludes the all-too-real possibility that some stocks might temporarily underperform over part of the investment horizon (in the sense that the mean return rate, $b_{n}(t)$, is less than the interest rate, $r(t)$, for some values of $t$ ), but perform well in the remainder of the trading interval. The preceding duality analysis removes these restrictions and works for completely general conical constraints.

\section{Utility maximization}

In this section we put aside the problem of mean-variance minimization considered in the previous sections and turn our attention to problems of utility maximization with convex portfolio constraints. Our goal is to demonstrate that the approach used for mean-variance minimization applies equally well to utility maximization, and thus constitutes a unified method of solving both of these problems. From now on we assume the following condition.

\section{Condition 7.1. We are given}

(i) a market with information filtration (2.1), a bond with price $\left\{S_{0}(t)\right\}$, and $N$ stocks with prices $\left\{S_{n}(t)\right\}, n=1,2, \ldots, N$, modelled as in Section 2 by the relations (2.2) and (2.3) and subject to Condition 2.1;

(ii) a closed, convex portfolio constraint set $K \subset \mathbb{R}^{N}$ with $\mathbf{0} \in K$;

(iii) an initial fortune $x_{0} \in(0, \infty)$; and

(iv) a utility function $U:(0, \infty) \rightarrow \mathbb{R}$ which is of class $C^{1}$, strictly increasing, strictly concave, and satisfies

(a) $\lim _{x \rightarrow \infty} U(x)=\infty$,

(b) $\lim _{x \rightarrow \infty} U^{(1)}(x)=0$,

(c) $\lim _{x \downarrow 0} U^{(1)}(x)=\infty$, and

(d) $\lim _{x \downarrow 0} U(x)>-\infty$.

For an $\left\{\mathcal{F}_{t}\right\}$-progressively measurable process $\pi: \Omega \times[0, T] \rightarrow \mathbb{R}^{N}$ such that

$$
\int_{0}^{T}\|\boldsymbol{\pi}(t)\|^{2} \mathrm{~d} t<\infty \quad \text { a.s. }
$$


let $\left\{X^{\pi}(t), t \in[0, T]\right\}$ be the unique $\mathbb{R}$-valued, continuous, $\left\{\mathcal{F}_{t}\right\}$-progressively measurable process determined by

$$
\mathrm{d} X^{\pi}(t)=X^{\pi}(t)\left\{r(t)+\pi^{\top}(t) \boldsymbol{\sigma}(t) \boldsymbol{\theta}(t)\right\} \mathrm{d} t+X^{\pi}(t) \boldsymbol{\pi}^{\top}(t) \boldsymbol{\sigma}(t) \mathrm{d} \boldsymbol{W}(t), \quad X^{\pi}(0)=x_{0} .
$$

Then $X^{\pi}$ is P-strictly positive, that is, $\inf _{t \in[0, T]} X^{\pi}(t)>0$ a.s., and if $\pi_{n}(t)$, the $n$th entry of $\boldsymbol{\pi}(t)$, is interpreted as the fraction of a small investor's total wealth put into the stock with price $S_{n}(t)$ (as is customary in problems of constrained utility maximization), then $X^{\pi}(t)$ gives the investor's total wealth at instant $t$, provided that the investor follows a self-funded strategy (see [5, p. 770]). Define the set of admissible portfolios,

$$
\begin{aligned}
\mathcal{A}^{\prime}:=\left\{\pi: \Omega \times[0, T] \rightarrow \mathbb{R}^{N}\right. \text { such that } \\
\left.\qquad \pi \in \mathcal{F}^{*}, \boldsymbol{\pi}(t) \in K \text { a.e., and } \int_{0}^{T}\|\boldsymbol{\pi}(t)\|^{2} \mathrm{~d} t<\infty \text { a.s. }\right\}
\end{aligned}
$$

(recall $\mathcal{F}^{*}$ as defined in Section 3), and the value of the portfolio optimization problem,

$$
\vartheta:=\sup _{\pi \in \mathcal{A}^{\prime}} \mathrm{E}\left[U\left(X^{\pi}(T)\right)\right]
$$

To avoid trivialities, assume that $\vartheta \in \mathbb{R}$. The utility maximization problem is to

$$
\text { establish the existence of some } \bar{\pi} \in \mathcal{A}^{\prime} \text { such that } \vartheta=\mathrm{E}\left[U\left(X^{\bar{\pi}}(T)\right)\right] \text {. }
$$

Remark 7.1. In contrast to the problem of mean-variance minimization considered in the preceding sections, the optimal wealth process for the utility maximization problem is generally not square integrable when $\pi \in \mathcal{A}^{\prime}$; hence, the set $\mathbb{B}$ of Section 3 is not the appropriate one in which to embed the utility maximization problem. Instead, we introduce the set $\mathbb{I}$ of all $\left\{\mathcal{F}_{t}\right\}$-Itô processes $\{X(t), t \in[0, T]\}$ of the form (3.1) for some (a.e. unique) $\left\{\mathcal{F}_{t}\right\}$-progressively measurable mappings $\dot{X}: \Omega \times[0, T] \rightarrow \mathbb{R}$ and $\Lambda_{X}: \Omega \times[0, T] \rightarrow \mathbb{R}^{N}$ such that $\int_{0}^{T}|\dot{X}(t)| \mathrm{d} t<$ $\infty$ and $\int_{0}^{T}\left\|\boldsymbol{\Lambda}_{X}(t)\right\|^{2} \mathrm{~d} t<\infty$ a.s., and write $X \equiv\left(X_{0}, \dot{X}, \boldsymbol{\Lambda}_{X}\right) \in \mathbb{I}$ to indicate that $X_{0} \in \mathbb{R}, \dot{X}$, and $\boldsymbol{\Lambda}_{X}$ satisfy these a.s. bounds and that (3.1) holds.

By analogy with (5.3), and recalling (7.1) and (7.2), let

$$
\begin{aligned}
\mathcal{C}(X):=\left\{\boldsymbol{\pi} \in \mathcal{A}^{\prime}: \dot{X}(t)=X(t)\left\{r(t)+\boldsymbol{\pi}^{\top}(t) \boldsymbol{\sigma}(t) \boldsymbol{\theta}(t)\right\}\right. \\
\left.\quad \text { and } \boldsymbol{\Lambda}_{X}(t)=X(t) \boldsymbol{\sigma}^{\top}(t) \boldsymbol{\pi}(t) \text { a.e. }\right\}
\end{aligned}
$$

for each $X \equiv\left(X_{0}, \dot{X}, \Lambda_{X}\right) \in \mathbb{I}$. Then, by an argument identical to that yielding (5.4), we have

$$
\vartheta=\sup _{\substack{X \in \mathbb{I}, X_{0}=x_{0} \\ \mathcal{C}(X) \neq \varnothing}} \mathrm{E}[U(X(T))] .
$$

We now introduce penalty functions for the constraints in (7.6) (much as we did for those in (5.4)). From Remark 2.1, (7.2), and (7.5) we see that, for each $X \equiv\left(X_{0}, \dot{X}, \Lambda_{X}\right) \in \mathbb{I}$ with $X_{0}>0$,

$$
\begin{gathered}
\mathcal{C}(X) \neq \varnothing \quad \Longleftrightarrow \quad \inf _{t \in[0, T]} X(t)>0 \text { a.s., } \quad \dot{X}(t)=r(t) X(t)+\boldsymbol{\Lambda}_{X}^{\top}(t) \boldsymbol{\theta}(t), \\
\text { and } X^{-1}(t)\left[\boldsymbol{\sigma}^{\top}\right]^{-1}(t) \boldsymbol{\Lambda}_{X}(t) \in K \text { a.e. }
\end{gathered}
$$


Remark 7.2. If $\mathcal{C}(X) \neq \varnothing$ for $X \equiv\left(X_{0}, \dot{X}, \boldsymbol{\Lambda}_{X}\right) \in \mathbb{I}$ with $X_{0}>0$, then $X^{-1}\left[\boldsymbol{\sigma}^{\top}\right]^{-1} \boldsymbol{\Lambda}_{X} \in$ $\mathcal{C}(X)$.

Motivated by (7.7), we define the mapping $L: \Omega \times[0, T] \times \mathbb{R} \times \mathbb{R} \times \mathbb{R}^{N} \rightarrow\{0, \infty\}$ by $L(\omega, t, x, v, \boldsymbol{\xi}):= \begin{cases}0 & \text { if } x>0, v=r(\omega, t) x+\boldsymbol{\xi}^{\top} \boldsymbol{\theta}(\omega, t), \text { and } x^{-1}\left[\boldsymbol{\sigma}^{\top}\right]^{-1}(\omega, t) \xi \in K, \\ \infty & \text { otherwise }\end{cases}$

(cf. (5.6)). Next, define $l_{0}(x)$ as in (5.8), let

$$
l_{T}(x):= \begin{cases}-U(x) & \text { if } x \in(0, \infty) \\ \infty & \text { otherwise }\end{cases}
$$

and define the mappings $m_{0}(\cdot), m_{T}(\cdot)$, and $M(\cdot)$ as in (5.12) (with $l_{0}(\cdot), l_{T}(\cdot)$, and $L(\cdot)$ as given in (5.8), (7.9), and (7.8), suppressing $\omega$ in the second relation of (5.12)). Then

$$
m_{0}(y)=x_{0} y, \quad m_{T}(y)=\tilde{U}(y):=\sup _{x>0}\{U(x)-x y\}, \quad y \in \mathbb{R},
$$

and an easy calculation based on (7.8), (5.15), and (5.12) shows that

$$
M(\omega, t, y, s, \boldsymbol{\gamma})= \begin{cases}0 & \text { for } s+r(\omega, t) y+\delta(-\boldsymbol{\sigma}(\omega, t)[\boldsymbol{\theta}(\omega, t) y+\boldsymbol{\gamma}]) \leq 0 \\ \infty & \text { otherwise }\end{cases}
$$

for each $(\omega, t, y, s, \boldsymbol{\gamma}) \in \Omega \times[0, T] \times \mathbb{R} \times \mathbb{R} \times \mathbb{R}^{N}$.

Remark 7.3. For $X \equiv\left(X_{0}, \dot{X}, \boldsymbol{\Lambda}_{X}\right) \in \mathbb{I}$ and $Y \equiv\left(Y_{0}, \dot{Y}, \boldsymbol{\Lambda}_{Y}\right) \in \mathbb{I}$, we shall continue to use the notation $\mathbb{M}(X, Y)(t)$ introduced in the statement of Proposition 5.1 and the notation $\boldsymbol{\Theta}_{Y}(t)$ introduced in (5.23). In this case, it follows at once from Itô's formula that $\left\{\left(\mathbb{M}(X, Y)(t), \mathcal{F}_{t}\right), t \in[0, T]\right\}$ is a continuous local martingale with $\mathbb{M}(X, Y)(0)=0$ but is not necessarily a genuine martingale. It follows that we can no longer avail ourselves of Proposition 5.1 in constructing the dual problem (as we did in Proposition 5.2). In order to deal with this we define

$$
\begin{aligned}
& \mathbb{I}_{1}:=\left\{X \in \mathbb{I}: X_{0}=x_{0}, \mathcal{C}(X) \neq \varnothing\right\}, \\
& \mathbb{I}_{2}:=\left\{Y \in \mathbb{I}: \dot{Y}(t)+r(t) Y(t)+\delta\left(\Theta_{Y}(t)\right) \leq 0, Y(t) \geq 0 \text { a.e. }\right\}
\end{aligned}
$$

from (5.8), (7.7), (7.8), and (7.11), for each $(X, Y) \in \mathbb{I}_{1} \times \mathbb{I}_{2}$ we then have

$$
l_{0}\left(X_{0}\right)=0, \quad L\left(t, X(t), \dot{X}(t), \boldsymbol{\Lambda}_{X}(t)\right)=0, \quad \text { and } \quad M\left(t, Y(t), \dot{Y}(t), \boldsymbol{\Lambda}_{Y}(t)\right)=0 \quad \text { a.e. }
$$

This, together with the third relation of (5.12), ensures that, for each $(X, Y) \in \mathbb{I}_{1} \times \mathbb{I}_{2}$,

$$
\begin{aligned}
0 & =L\left(t, X(t), \dot{X}(t), \boldsymbol{\Lambda}_{X}(t)\right)+M\left(t, Y(t), \dot{Y}(t), \boldsymbol{\Lambda}_{Y}(t)\right) \\
& \geq X(t) \dot{Y}(t)+\dot{X}(t) Y(t)+\boldsymbol{\Lambda}_{X}^{\top}(t) \boldsymbol{\Lambda}_{Y}(t) \quad \text { a.e., }
\end{aligned}
$$

and we thus find that $\mathbb{M}(X, Y)(t) \geq X(t) Y(t)-X_{0} Y_{0}$ a.e. for each $(X, Y) \in \mathbb{I}_{1} \times \mathbb{I}_{2}$. Since $X(t) Y(t) \geq 0$ a.e. for each $(X, Y) \in \mathbb{I}_{1} \times \mathbb{I}_{2}$ (by (7.7), (7.12), and (7.13)), it follows that $\mathbb{M}(X, Y)(t) \geq-X_{0} Y_{0}$ a.e. and, thus, from Remark 7.3 and Fatou's lemma, that

$\left\{\left(\mathbb{M}(X, Y)(t), \mathcal{F}_{t}\right), t \in[0, T]\right\} \quad$ is a supermartingale with $\quad \mathbb{M}(X, Y)(0)=0$,

$$
\text { for each }(X, Y) \in \mathbb{I}_{1} \times \mathbb{I}_{2} \text {. }
$$


Remark 7.4. We now define $\Phi(X)$ for each $X \in \mathbb{I}_{1}$ and $\Psi(Y)$ for each $Y \in \mathbb{I}_{2}$ as in (5.9) and (5.16), respectively, with $l_{0}, l_{T}$, and $L$ as given in (5.8), (7.9), and (7.8), respectively, and with $m_{0}, m_{T}$, and $M$ as given in (7.10) and (7.11), respectively. Then, from (7.14), we have

$$
\Phi(X)=-\mathrm{E}[U(X(T))], \quad \Psi(Y)=x_{0} Y_{0}+\mathrm{E}[\tilde{U}(Y(T))], \quad(X, Y) \in \mathbb{I}_{1} \times \mathbb{I}_{2} .
$$

Proposition 7.1. Assume that Condition 7.1 is satisfied and that $\vartheta \in \mathbb{R}$ (see (7.3)). Then $\Phi(X)>-\infty$ and $\Psi(Y)>-\infty$ for each $(X, Y) \in \mathbb{I}_{1} \times \mathbb{I}_{2}$ (recall Remark 7.4), and

$$
\Phi(X)+\Psi(Y) \geq 0, \quad(X, Y) \in \mathbb{I}_{1} \times \mathbb{I}_{2} .
$$

Moreover, for arbitrary $\bar{X} \equiv\left(\bar{X}_{0}, \dot{\bar{X}}, \boldsymbol{\Lambda}_{\bar{X}}\right) \in \mathbb{I}_{1}$ and $\bar{Y} \equiv\left(\bar{Y}_{0}, \dot{\bar{Y}}, \boldsymbol{\Lambda}_{\bar{Y}}\right) \in \mathbb{I}_{2}$, we have $\Phi(\bar{X})+$ $\Psi(\bar{Y})=0$ if and only if each of the following conditions hold:

$$
\begin{gathered}
l_{0}\left(\bar{X}_{0}\right)+m_{0}\left(\bar{Y}_{0}\right)=\bar{X}_{0} \bar{Y}_{0}, \\
l_{T}(\bar{X}(T))+m_{T}(\bar{Y}(T))=-\bar{X}(T) \bar{Y}(T) \quad \text { a.s. }, \\
L\left(t, \bar{X}(t), \dot{\bar{X}}(t), \boldsymbol{\Lambda}_{\bar{X}}(t)\right)+M\left(t, \bar{Y}(t), \dot{\bar{Y}}(t), \boldsymbol{\Lambda}_{\bar{Y}}(t)\right) \\
=\bar{X}(t) \dot{\bar{Y}}(t)+\dot{\bar{X}}(t) \bar{Y}(t)+\Lambda_{\bar{X}}^{\top}(t) \Lambda_{\bar{Y}}(t) \quad \text { a.e. }, \\
\{\bar{X}(t) \bar{Y}(t), t \in[0, T]\} \quad \text { is an }\left\{\mathcal{F}_{t}\right\} \text {-martingale. }
\end{gathered}
$$

Proof. Fix a pair $(X, Y) \in \mathbb{I}_{1} \times \mathbb{I}_{2}$. That $\Phi(X)>-\infty$ and $\Psi(Y)>-\infty$ is an immediate consequence of Condition 7.1(iv) and that fact that $\vartheta \in \mathbb{R}$. From the definitions of $\Phi(X)$ and $\Psi(Y)$ in Remark 7.4, we see that the chain of equalities and inequalities in (5.22) continues to hold. Moreover, $\mathrm{E}[-\mathbb{M}(X, Y)(T)] \geq 0$ (from (7.16)), and (7.18) follows from this and (5.22). Next suppose that $\Phi(\bar{X})+\Psi(\bar{Y})=0$ for some $(\bar{X}, \bar{Y}) \in \mathbb{I}_{1} \times \mathbb{I}_{2}$. Then, since $\mathrm{E}[-\mathbb{M}(\bar{X}, \bar{Y})(T)] \geq 0$ (again from (7.16)), the inequality in (5.22) must be an equality (with $(\bar{X}, \bar{Y})$ in place of $(X, Y))$ and we must have $\mathrm{E}[\mathbb{M}(\bar{X}, \bar{Y})(T)]=0$. Equations (7.19)-(7.21) follow from this and the general relations in (5.21), and it follows from (7.16) that $\{\mathbb{M}(\bar{X}, \bar{Y})(t)\}$ is actually an $\left\{\mathscr{F}_{t}\right\}$-martingale (being a supermartingale with constant expectation). However, (7.21) together with (7.14) give $\mathbb{M}(\bar{X}, \bar{Y})(t)=\bar{X}(t) \bar{Y}(t)-\bar{X}_{0} \bar{Y}_{0}$, which establishes (7.22). The converse, that (7.19)-(7.22) imply that $\Phi(\bar{X})+\Psi(\bar{Y})=0$, is immediate from (7.14) and (5.22).

Remark 7.5. Condition 7.1 ensures that $\tilde{U}(\cdot)$ is smooth, and the derivative $U^{(1)}(\cdot)$ has a continuous, strictly decreasing inverse $I:(0, \infty) \rightarrow(0, \infty)$ with $I(y)=-\tilde{U}^{(1)}(y), y \in$ $(0, \infty)$ (see [9, Lemma 3.4.3]). From (7.9) and (7.10), for each $x, y \in \mathbb{R}$ we have the equivalence

$$
l_{T}(x)+m_{T}(y)=-x y \quad \Longleftrightarrow \quad y \in(0, \infty), \quad x=I(y) \in(0, \infty) .
$$

Similarly, from (5.8) and (7.10), for each $x, y \in \mathbb{R}$ we have $l_{0}(x)+m_{0}(y)=x y$ if and only if $x=x_{0}$.

Proposition 7.2. Suppose that the conditions of Proposition 7.1 are satisfied. Then, for an arbitrary $(\bar{X}, \bar{Y}) \in \mathbb{I}_{1} \times \mathbb{I}_{2}$, we have (recalling $\boldsymbol{\Theta}_{Y}(\cdot)$, $\vartheta$, and $\delta(\cdot)$ as respectively defined in Remark 7.3, (7.3), and (5.15))

$$
\mathrm{E}[U(\bar{X}(T))]=\vartheta=\inf _{Y \in \mathbb{I}_{2}} \Psi(Y)=\Psi(\bar{Y})
$$


if and only if

$$
\begin{gathered}
\bar{X}_{0}=x_{0}, \\
\bar{Y}(T)>0 \quad \text { and } \quad \bar{X}(T)=I(\bar{Y}(T))>0 \text { a.s. } \\
\dot{\bar{Y}}(t)+r(t) \bar{Y}(t)+\delta\left(\boldsymbol{\Theta}_{\bar{Y}}(t)\right)=0 \text { a.e. }, \\
\bar{\pi} \in \mathcal{C}(\bar{X}) \quad \text { and } \delta\left(\boldsymbol{\Theta}_{\bar{Y}}(t)\right)+\bar{\pi}^{\top}(t) \boldsymbol{\Theta}_{\bar{Y}}(t)=0 \text { a.e. } \\
\text { for } \overline{\boldsymbol{\pi}}(t):=\bar{X}^{-1}(t)\left[\sigma^{\top}\right]^{-1}(t) \boldsymbol{\Lambda}_{\bar{X}}(t), \\
\{\bar{X}(t) \bar{Y}(t), t \in[0, T]\} \quad \text { is an }\left\{\mathcal{F}_{t}\right\} \text {-martingale. }
\end{gathered}
$$

Proof. In view of (5.15), (7.8), and (7.11), we have the following equivalence: for arbitrary $(\omega, t) \in \Omega \times[0, T], x, y, v, s \in \mathbb{R}$, and $\boldsymbol{\xi}, \boldsymbol{\gamma} \in \mathbb{R}^{N}$,

$$
\begin{aligned}
& L(\omega, t, x, v, \xi)+M(\omega, t, y, s, \boldsymbol{\gamma})=x s+y v+\boldsymbol{\xi}^{\top} \boldsymbol{\gamma} \\
& \Longleftrightarrow \quad x>0, \quad v=r(t) x+\boldsymbol{\xi}^{\top} \boldsymbol{\theta}(t), \quad x^{-1}\left[\boldsymbol{\sigma}^{\top}\right]^{-1}(t) \xi \in K, \\
& s+y r(t)+\delta(-\boldsymbol{\sigma}(t)[\boldsymbol{\theta}(t) y+\boldsymbol{\gamma}])=0, \quad \text { and } \\
& \delta(-\boldsymbol{\sigma}(t)[\boldsymbol{\theta}(t) y+\boldsymbol{\gamma}])+x^{-1} \boldsymbol{\xi}^{\top} \boldsymbol{\sigma}^{-1}(t)(-\boldsymbol{\sigma}(t)[\boldsymbol{\theta}(t) y+\boldsymbol{\gamma}])=0 .
\end{aligned}
$$

Fix an arbitrary $(\bar{X}, \bar{Y}) \in \mathbb{I}_{1} \times \mathbb{I}_{2}$. In view of (7.29), (7.12), (7.7), Remark 7.5 and Remark 7.2, we find that (7.19)-(7.22) are equivalent to (7.24)-(7.28). Moreover, from (7.6), (7.12), and Remark 7.4 we have $\vartheta=\sup _{X \in \mathbb{I}_{1}}\{-\Phi(X)\}$, and, thus, the condition $\Phi(\bar{X})+\Psi(\bar{Y})=0$ is equivalent to (7.23), as follows from the weak duality (7.18). The equivalence of (7.23) and (7.24)-(7.28) now follows from Proposition 7.1.

Remark 7.6. It follows from Proposition 7.2 that solving problem (7.4) requires constructing a pair $(\bar{X}, \bar{Y}) \in \mathbb{I}_{1} \times \mathbb{I}_{2}$ which satisfies the relations (7.24)-(7.28), since then the optimal portfolio $\bar{\pi}$ is given in terms of $\bar{X}$ by (7.27). To this end we make the following observations.

(a) If $(\bar{X}, \bar{Y}) \in \mathbb{I}_{1} \times \mathbb{I}_{2}$ is a pair satisfying (7.24)-(7.28), then $\bar{Y}$ is necessarily P-strictly positive (since (7.25) ensures that $\bar{X}(T) \bar{Y}(T)>0$ a.s. and it thus follows from (7.28) and $[8$, Problem 1.3 .29$, p. 21] that $\bar{X} \bar{Y}$ is a P-strictly positive process, while (7.27) and (7.7) ensure that the process $\bar{X}$ is P-strictly positive). This, together with (7.26), shows that we need minimize $\Psi(\cdot)$, not over all of $\mathbb{I}_{2}$, but instead over the smaller set $\mathbb{I}_{3} \subset \mathbb{I}_{2}$ defined by

$$
\mathbb{I}_{3}:=\left\{Y \in \mathbb{I}: \inf _{t \in[0, T]} Y(t)>0 \text { a.s. and } \dot{Y}(t)+r(t) Y(t)+\delta\left(\boldsymbol{\Theta}_{Y}(t)\right)=0 \text { a.e. }\right\} .
$$

The advantage of minimizing over $\mathbb{I}_{3}$ is that the $Y \in \mathbb{I}_{3}$ are exponential semimartingales. In fact, with

$$
\mathcal{Q}:=\left\{\boldsymbol{v}: \Omega \times[0, T] \rightarrow \mathbb{R}^{N} \text { such that } \boldsymbol{v} \in \mathcal{F}^{*} \text { and } \int_{0}^{T}\left\{\|\boldsymbol{v}(t)\|^{2}+\delta(\boldsymbol{v}(t))\right\} \mathrm{d} t<\infty \text { a.s. }\right\}
$$

and

$$
H_{\nu}(t):=\exp \left[-\int_{0}^{t}\{r(\tau)+\delta(\boldsymbol{v}(\tau))\} \mathrm{d} \tau\right] \mathcal{E}\left(-\left[\boldsymbol{\theta}+\boldsymbol{\sigma}^{-1} \boldsymbol{v}\right]^{\top} \bullet \boldsymbol{W}\right)(t), \quad \boldsymbol{v} \in \mathbb{Q}
$$

(see Remark 5.8), it follows easily from Itô's formula that $\mathbb{I}_{3}=\left\{y H_{v}: y \in(0, \infty), \boldsymbol{v} \in \mathcal{Q}\right\}$. 
(b) Minimization of $\Psi(\cdot)$ over $\mathbb{I}_{3}$ is still difficult, because the set $\mathcal{Q}$ is 'very large'. Accordingly, we restrict our attention to $\boldsymbol{v} \in \mathscr{D}:=\left\{\boldsymbol{v} \in \mathbb{Q}: \mathrm{E}\left[\int_{0}^{T}\|\boldsymbol{v}(t)\|^{2} \mathrm{~d} t\right]<\infty\right\}$ (that is, squareintegrable members of $\mathcal{Q})$ and minimize $\Psi(\cdot)$ over $\mathbb{I}_{4}:=\left\{y H_{v}: y \in(0, \infty), \boldsymbol{v} \in \mathcal{D}\right\} \subset \mathbb{I}_{3}$. That is, we shall establish that $\Psi(\bar{Y})=\inf _{Y \in \mathbb{I}_{4}} \Psi(Y)$ for some $\bar{Y} \in \mathbb{I}_{4}$. To this end, in addition to Condition 7.1 we must assume that

- $x \mapsto x U^{(1)}(x)$ is nondecreasing on $(0, \infty)$,

- there exist $\gamma \in(1, \infty)$ and $\alpha \in(0,1)$ such that $\alpha U^{(1)}(x) \geq U^{(1)}(\gamma x)$ for all $x \in(0, \infty)$, and,

- for each $y \in(0, \infty)$, there exists some $v \in \mathscr{D}$ such that $\mathrm{E}\left[\tilde{U}\left(y H_{v}(T)\right)\right]<\infty$.

Using these conditions and [7, Proposition II.1.2] (which relies on the square integrability of $v \in D$ ), together with a trivial variant of the proof of [5, Proposition 13.2], we see that there exists a pair $(\bar{y}, \bar{v}) \in(0, \infty) \times \mathscr{D}$ such that $\inf _{Y \in \mathbb{I}_{4}} \Psi(Y)=\Psi(\bar{Y})$ for $\bar{Y}:=\bar{y} H_{\bar{v}} \in \mathbb{I}_{4}$ (this is analogous to Proposition 5.4). It remains to construct an $\bar{X} \in \mathbb{I}_{1}$ in terms of this $\bar{Y}$ such that (7.24)-(7.28) hold. Motivated by (7.25) and (7.28), we define

$$
\bar{X}(t):=\bar{Y}^{-1}(t) \operatorname{E}\left[\bar{Y}(T) I(\bar{Y}(T)) \mid \mathcal{F}_{t}\right]=H_{\bar{\nu}}^{-1}(t) \mathrm{E}\left[H_{\bar{\nu}}(T) I\left(\bar{y} H_{\bar{\nu}}(T)\right) \mid \mathcal{F}_{t}\right] .
$$

From (7.32) we have $\bar{X}(t) H_{\bar{v}}(t)=\bar{X}(0)+\int_{0}^{t} \overline{\boldsymbol{\psi}}^{\top}(\tau) \mathrm{d} \boldsymbol{W}(\tau)=: \xi_{0}(t)$, for some $\mathbb{R}^{N}$-valued, a.e. unique $\bar{\psi} \in \mathcal{F}^{*}$ with $\int_{0}^{T}\|\bar{\psi}(t)\|^{2} \mathrm{~d} t<\infty$ a.s. (by the martingale representation theorem). Using this, together with (7.31), to expand the quotient $\bar{X}(t)=\xi_{0}(t) / H_{\bar{\nu}}(t)$ using Itô's formula then gives $\bar{X} \in \mathbb{I}$ (recall Remark 7.1) with $\{\dot{\bar{X}}(t)\}$ and $\left\{\boldsymbol{\Lambda}_{\bar{X}}(t)\right\}$ given by

$$
\mathrm{d} \bar{X}(t)=\bar{X}(t)\left\{r(t)+\overline{\boldsymbol{\pi}}^{\top}(t) \boldsymbol{\sigma}(t) \boldsymbol{\theta}(t)+\delta(\overline{\boldsymbol{v}}(t))+\overline{\boldsymbol{\pi}}^{\top}(t) \overline{\boldsymbol{v}}(t)\right\} \mathrm{d} t+\bar{X}(t) \overline{\boldsymbol{\pi}}^{\top}(t) \boldsymbol{\sigma}(t) \mathrm{d} \boldsymbol{W}(t)
$$

for

$$
\overline{\boldsymbol{\pi}}(t):=\left[\boldsymbol{\sigma}^{\top}\right]^{-1}(t)\left[H_{\bar{v}}^{-1}(t) \bar{X}^{-1}(t) \overline{\boldsymbol{\psi}}(t)+\boldsymbol{\theta}(t)+\boldsymbol{\sigma}^{-1}(t) \overline{\boldsymbol{v}}(t)\right]
$$

(cf. (5.41), (5.42), and (5.43)).

(c) From (7.32) and (7.30) we see that the pair $(\bar{X}, \bar{Y}) \in \mathbb{I} \times \mathbb{I}_{4}$ satisfies (7.25), (7.26), and (7.28). It remains to show that $\bar{X} \in \mathbb{I}_{1}$ and that (7.24) and (7.27) hold. To this end we use necessary conditions resulting from the optimality of $\bar{Y} \equiv \bar{y} H_{\bar{v}}$ established in (b). From this optimality, together with (7.17), we find that $x_{0} y+\mathrm{E}\left[\tilde{U}\left(y H_{v}(T)\right)\right] \geq x_{0} \bar{y}+\mathrm{E}\left[\tilde{U}\left(\bar{y} H_{\bar{v}}(T)\right)\right]$ for all $(y, v) \in$ $(0, \infty) \times \mathscr{D}$. In particular, $\bar{y}$ minimizes the function $y \mapsto x_{0} y+\mathrm{E}\left[\tilde{U}\left(y H_{\bar{v}}(T)\right)\right]$, and, upon taking the derivative in $y$ and using the identity $\tilde{U}^{(1)}(y)=-I(y)$ (see Remark 7.5), we obtain $x_{0}=\mathrm{E}\left[H_{\bar{v}}(T) I\left(\bar{y} H_{\bar{v}}(T)\right)\right]=\bar{X}(0)$, where the second equality follows from (7.32); this verifies (7.24). Again from the optimality established in (b), we also obtain $\mathrm{E}\left[\tilde{U}\left(\bar{y} H_{v}(T)\right)\right] \geq$ $\mathrm{E}\left[\tilde{U}\left(\bar{y} H_{\bar{v}}(T)\right)\right]$ for all $\boldsymbol{v} \in \mathcal{D}$, that is, $(1 / \varepsilon) \mathrm{E}\left[\tilde{U}\left(\bar{y} H_{\bar{v}+\varepsilon \eta}(T)\right)-\tilde{U}\left(\bar{y} H_{\bar{v}}(T)\right)\right] \geq 0$ for each $\varepsilon \in(0,1)$ and $\eta \in \mathcal{D}$. Evaluating the limit as $\varepsilon \rightarrow 0$ for suitable choices of $\eta \in \mathcal{D}$ (which is a calculation essentially identical to that in [5, pp. 781-783], we obtain $\overline{\boldsymbol{\pi}}(t) \in K$ and $\delta(\overline{\boldsymbol{v}}(t))+\overline{\boldsymbol{\pi}}^{\top}(t) \overline{\boldsymbol{v}}(t)=0$ a.e. It follows from this, together with (7.33) and (7.5), that $\overline{\boldsymbol{\pi}} \in \mathcal{C}(\bar{X})$; thus, $\bar{X} \in \mathbb{I}_{1}$. Moreover, it is clear that $\boldsymbol{\Theta}_{\bar{Y}}(t)=\bar{Y}(t) \overline{\boldsymbol{v}}(t)$ for $\bar{Y}=\bar{y} H_{\bar{v}}$ and, thus, $\delta\left(\boldsymbol{\Theta}_{\bar{Y}}(t)\right)+\overline{\boldsymbol{\pi}}^{\top}(t) \boldsymbol{\Theta}_{\bar{Y}}(t)=0$ a.e. (since $\bar{Y}(t)>0$ and $\delta(\cdot)$ is positively homogeneous), as required to verify (7.27).

Remark 7.7. In order to focus on just the essential ideas, we have considered maximization of utility from terminal wealth only, without utility from consumption. Only a straightforward 
modification of the the preceding approach is necessary to include intertemporal consumption (see [11]).

Remark 7.8. The approach to problems of constrained utility maximization used in this section contrasts with those of [4], [5], and [10], which rely on the a-priori introduction of a complete fictitious market in which the money market rate and mean return rate on stocks are such that unconstrained utility maximization in the fictitious market amounts to constrained utility maximization in the given market. The approach of this section avoids fictitious markets (the formulation of which is not at all simple) and proceeds algorithmically by relying on elementary convex analysis to synthesize optimality relations (see (7.24)-(7.28)) the solution of which yields the optimal portfolio. The same approach establishes the existence of optimal portfolios for problems of mean-variance minimization. In cases of genuine practical interest this optimal portfolio is explicitly computable (see Example 6.2). Finally, while methods based on the introduction of a complete fictitious market are undoubtedly effective for problems of constrained utility maximization, provided that one can find the 'correct' fictitious market (always a significant challenge), this approach does not appear to adapt easily to constrained mean-variance minimization. On the other hand, methods of stochastic Riccati equations and stochastic linear quadratic control, which are the preferred mathematical technology in problems of mean-variance minimization, are unlikely to be appropriate for preferences based on utility maximization, relying as they do in an essential way on the quadratic form of the loss function. In contrast, the approach of this work applies with equal facility to both of the two main preference structures of utility maximization and mean-variance reduction, and deals easily with portfolio constraints.

Example 7.1. We add an induced constraint on the terminal wealth to the problem (7.4). Fix some $\alpha \in[0,1)$, define $\zeta:=\alpha x_{0} S_{0}(T)$, and let $\mathcal{A}^{*}:=\left\{\pi \in \mathcal{A}^{\prime}: X^{\pi}(T) \geq \zeta\right.$ a.s. $\}$ (recall (7.2)) and $\vartheta_{1}:=\sup _{\pi \in \mathcal{A}^{*}} \mathrm{E}\left[U\left(X^{\pi}(T)\right)\right]$. The problem of utility maximization that we study is to establish the existence of some $\bar{\pi} \in \mathcal{A}^{*}$ such that $\vartheta_{1}=\mathrm{E}\left[U\left(X^{\bar{\pi}}(T)\right)\right]$. This problem could represent the preference of a cautious investor whose goal is to maximize expected utility from terminal wealth subject to the usual portfolio constraints while also insisting that the terminal wealth not be less than the fortune, $\zeta$, that would have been obtained by just investing some fraction $\alpha$ of the initial wealth, $x_{0}$, in a money market account. While it is difficult to use the method of fictitious markets to establish optimality relations for this problem, we shall see that the approach of this section applies quite easily. Define a modified utility by $U_{1}(\omega, x):=U(x)$ for $x \geq \zeta(\omega)$ and $U_{1}(\omega, x):=-\infty$ otherwise, and let $\tilde{U}_{1}(\omega, y):=\sup _{x>0}\left\{U_{1}(\omega, x)-x y\right\}$ and $I_{1}(\omega, y):=-\tilde{U}_{1}^{(1)}(\omega, y)$ for all $(\omega, y) \in \Omega \times(0, \infty)$. Then, exactly as in (7.6), we find that $\vartheta_{1}=\sup \left\{\mathrm{E}\left[U_{1}(X(T))\right]: X \in \mathbb{I}, X_{0}=x_{0}, \mathcal{C}(X) \neq \varnothing\right\}$. Finally, by analogy with (7.17), let $\Phi_{1}(X):=-\mathrm{E}\left[U_{1}(X(T))\right]$ for each $X \in \mathbb{I}_{1}$ and $\Psi_{1}(Y):=x_{0} Y_{0}+\mathrm{E}\left[\tilde{U}_{1}(Y(T))\right]$ for each $Y \in \mathbb{I}_{2,1}:=\left\{Y \in \mathbb{I}_{2}: \mathrm{E}[Y(T)]<\infty\right\}$. We can now repeat the analysis which led to Proposition 7.2, but using the utility function $U_{1}$ in place of $U$, to show that, for arbitrary $(\bar{X}, \bar{Y}) \in \mathbb{I}_{1} \times \mathbb{I}_{2,1}$, we have $\mathrm{E}\left[U_{1}(\bar{X}(T))\right]=\vartheta_{1}=\inf _{Y \in \mathbb{I}_{2,1}} \Psi_{1}(Y)=\Psi_{1}(\bar{Y})$ if and only if the Euler-Lagrange and transversality relations (7.24), (7.26)-(7.28), and

$$
\bar{Y}(T)>0 \quad \text { and } \quad \bar{X}(T)=I_{1}(\omega, \bar{Y}(T)) \text { a.s. }
$$

hold (that is, we have (7.34) in place of the transversality relation (7.25)). It is now necessary to solve these relations, exactly as in Remark 7.6. For the sake of simplicity we look at the special case in which $K=\mathbb{R}^{N}$. Then, just as in Example 6.1, the dual problem reduces to the minimization of the functional $y \mapsto \Psi_{1}(y H):(0, \infty) \rightarrow \mathbb{R}$ (recall (5.39)). It is clear 
from the definition of $\tilde{U}$ that $\lim _{y \downarrow 0} \Psi_{1}(y H)=\infty$ and, since $\alpha<1$, it is easily verified that $\lim _{y \rightarrow \infty} \Psi_{1}(y H)=\infty$, so the existence of a minimizing $\bar{y} \in(0, \infty)$ follows. Now define $\vec{Y}(t):=\bar{y} H(T)$ and $\bar{X}(t):=H^{-1}(t) \mathrm{E}\left[H(T) I_{1}(\bar{y} H(T)) \mid \mathcal{F}_{t}\right]$. Then it is clear that $(\bar{X}, \bar{Y}) \in \mathbb{I}_{1} \times \mathbb{I}_{2,1}$, and, just as in Remark 7.6, it can be established that this pair satisfies the relations (7.24), (7.26)-(7.28), and (7.34). It now follows that $\overline{\boldsymbol{\pi}}$ defined in terms of $\bar{X}$ by (7.27) is the optimal portfolio. When the constraint on the terminal wealth binds, we can use the fact that $\alpha<1$ to relax the dual problem (by essentially following the approach of [6]) in order to establish the existence of a Lagrange multiplier in $L^{\infty}(\Omega, \mathcal{F}, \mathrm{P})^{*}$ (the topological dual of $\left.L^{\infty}(\Omega, \mathcal{F}, \mathrm{P})\right)$ which enforces the terminal wealth constraint.

\section{References}

[1] Aubin, J.-P. (1978). Applied Functional Analysis. John Wiley, New York.

[2] Bismut, J.-M. (1973). Conjugate convex functions in optimal stochastic control. J. Math. Anal. Appl. 44, 384404.

[3] Chow, Y. S. And TeIcher, H. (1988). Probability Theory: Independence, Interchangeability, Martingales, 2nd edn. Springer, New York.

[4] Cuoco, D. AND LiU, H. (2000). A martingale characterization of consumption choices and hedging costs with margin requirements. Math. Finance 10, 355-385.

[5] Cvitanić, J. and Karatzas, I. (1992). Convex duality in constrained portfolio optimization. Ann. Appl. Prob. 2, 767-818.

[6] Dubovitskit, A. YA. And Mil'yutin, A. A. (1968). Necessary conditions for a weak extremum in problems of optimal control with mixed inequality constraints. Zhur. Vychislitel. Mat. Mat. Fys. 8, 725-779.

[7] Ekeland, I. and Témam, R. (1999). Convex Analysis and Variational Problems (Classics Appl. Math. 88). Society for Industrial and Applied Mathematics, Philadelphia, PA.

[8] Karatzas, I. And Shreve, S. E. (1988). Brownian Motion and Stochastic Calculus. Springer, New York.

[9] Karatzas, I. And Shreve, S. E. (1998). Methods of Mathematical Finance. Springer, New York.

[10] Karatzas, I., Lehoczky, J. P., Shreve, S. E. And Xu, G. L. (1991). Martingale and duality methods for utility maximization in an incomplete market. SIAM J. Control Optimization 29, 702-730.

[11] LABBÉ, C. (2004). Contributions to the theory of constrained portfolio optimization. Doctoral Thesis, Department of Statistics and Actuarial Sciences, University of Waterloo.

[12] Li, X., Zhou, X. Y. And Lim, A. E. B. (2002). Dynamic mean-variance portfolio selection with no-shorting constraints. SIAM J. Control Optimization 40, 1540-1555.

[13] Lim, A. E. B. AND Zhou, X. Y. (2002). Mean-variance portfolio selection with random parameters in a complete market. Math. Operat. Res. 27, 101-120.

[14] Rogers, L. C. G. (2003). Duality in constrained optimal investment and consumption problems: a synthesis. In Paris-Princeton Lectures on Mathematical Finance, 2002 (Lecture Notes Math. 1814), Springer, Berlin, pp. $95-131$.

[15] Vinter, R. B. (2000). Optimal Control. Birkhäuser, Boston, MA.

[16] Yong, J. And Zhou, X. Y. (1999). Stochastic Controls: Hamiltonian Systems and HJB Equations. Springer, New York. 